\title{
Automated Assessment of Polyethylene Wear in Cemented Acetabular Components using Anteroposterior Radiographs of Total Hip Replacements
}

\author{
C. Stuart Kerrigan ${ }^{a}$ Stephen J. McKenna ${ }^{a}{ }^{*}$ Ian W. Ricketts ${ }^{a}$ \\ Carlos Wigderowitz ${ }^{\mathrm{b}}$

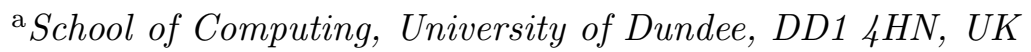 \\ b Orthopaedics and Trauma Surgery, Ninewells Hospital, Dundee, DD1 9SY, UK
}

\begin{abstract}
Polyethylene wear in the acetabular components of hip prostheses is implicated in loosening and failure. Radiographic measurement of wear is used to identify patients at risk and to assess prosthesis designs. This paper focuses on analysis of prostheses with cemented acetabular cups from anteroposterior radiographs. The articular surface of the femoral head and the acetabular rim marker are modelled as spherical and circular respectively, resulting in elliptical image projections. Methods for automatically localising these structures in radiographs are presented using robust ellipse fitting and various error functions. Special attention is paid to the acetabular marker since this often projects as a highly eccentric ellipse. Robust fitting enables successful localisation in the presence of clutter without the need for user interaction. Finally, the use of these ellipses as reference structures for wear estimation is investigated and the effect of eccentricity errors is highlighted.
\end{abstract}

\section{Introduction}

Acetabular wear is the major cause of aseptic loosening of total hip replacements (THR), leading to costly revision surgery and patient discomfort. Accurate estimation of wear is therefore important for early assessment of prosthesis performance. Wire markers are typically attached to the polyethylene acetabular component of a prosthesis so that both it and the metal femoral head component can be imaged effectively using standard x-ray imaging. Displacement over time of the femoral head component relative to the acetabular cup indicates wear, the main component of which will be apparent in an anteroposterior (AP) radiograph. In this paper, automated methods for measuring acetabular wear based on localising the femoral head and acetabular cup rim in AP radiographs are proposed and

* Corresponding author. Tel.: +44 1382 384732; fax: +44 1382385509. 
investigated. Both of these prosthesis components give rise to elliptical image structures. A contribution of this paper is to show how these structures can be reliably segmented using local models of appearance, local search, appropriate error functions and a statistically robust fitting algorithm. The use of relative displacement of these ellipses for wear measurement is investigated with particular emphasis on eccentricity errors which arise due to the highly eccentric appearance of the acetabular rim.

The remainder of the paper is organised as follows. Section 2 provides relevant background on total hip replacements. Section 3 briefly reviews existing methods for estimating acetabular wear. Section 4 describes the method used to model the shape and local appearance of the femoral head and acetabular rim marker in the image. Section 5 describes how these models can be used to perform localisation. Section 6 investigates the use of robust ellipse fitting to enable localisation in the presence of clutter and presents empirical comparisons using both synthetic and clinical data. Section 7 discusses wear estimation and investigates the extent to which eccentricity errors need to be considered. Finally, some conclusions regarding clinical relevance and implications for related methods are drawn in Section 8.

\section{Background}

Total hip replacement is one of the most common orthopaedic procedures. It is most often performed on patients aged between 50 and 60 but also on younger patients as well as animals (1; 2). Recipients have usually suffered from severe osteoarthritis, irreparable hip fractures or aseptic necrosis. Each hip prothesis has a femoral and an acetabular component. The former is typically made from titanium or cobalt-chromium alloy and the latter from ultra-high molecular weight polyethylene with the option of a metal backing. Numerous new models are patented every year. Furnes et al. (3) reported that there were over 200 different implant combinations used in Norway in 1996. Within the UK, Murray et al. (4) noted that 19 different companies were manufacturing 62 different models of implant for primary THRs, most without results published in peer-reviewed journals.

Hip prostheses can be categorised according to the method of fixation used. In a cemented THR, the acetabular cup and the femoral stem are cushioned to the skeleton with a layer of polymethylmethacrylate bone cement. The acetabular cup is sometimes metal backed to allow the changing of worn polyethylene components without disrupting the cementbone interface. Alternatively, in cementless THR, the acetabular cup is press-fitted, held in place by the tightness of fit and screws. Cementless acetabular cups are always metal backed since bone tissue does not react well to direct contact with polyethylene or ceramic. Some surgeons use a hybrid in which one component is cemented while the other is cementless (5). Cemented THR remains the most popular method of fixation. The experiments reported here used cemented acetabular cups with wire rim markers.

A THR is said to have failed when it becomes ineffective, typically when the patient experiences increased pain and loss of motion. The lifespan of a prosthesis is $10-20$ years (6) . In the UK, 5,000 of the 40,000 THRS performed annually are revisions (1). Revision THRs have a higher rate of infection and poorer performance (7). Aseptic loosening is the chief 
cause of THR failure and is related to prosthesis migration and wear of the acetabular component. For example, 75.3\% of revisions in Sweden during the period 1979-2000 were due to aseptic loosening (8). One recent study reported that out of 100 prostheses, 16 acetabular cups were revised due to osteolysis induced loosening (1).

Loosening can be caused by osteolysis, a condition in which bone resorption exceeds bone formation, resulting in loss of bone volume. McKellop et al. (9) suggest that osteolysis in THR patients is due to adhesion, abrasion and fatigue creating wear particles. These are less than $10 \mu \mathrm{m}$ in size and potentially consist of metal, ceramic, cement or polyethylene. Some particulate debris can penetrate the interface between prosthesis and bone, stimulating the cellular response leading to osteolysis (10). It was once thought that cement particles caused the lytic process and this motivated the introduction of cementless THR. However, femoral lysis is in fact more prominent with cementless components, suggesting that cement might actually protect against lysis (11). Acetabular cup polyethylene, the softest of the materials used in THR, is the major source of particulate debris. Attempts to reduce the rate of polyethylene wear have led to the creation of more durable acetabular cups. Cates et al. (12) noted that metal backing in cemented THRs, intended to stiffen the polyethylene and produce a more even distribution of stress, led to an increased rate of wear. More success has been obtained by crosslinking polyethylene carbon-hydrogen chains (9). Ceramic components manufactured from alumina and zirconia are smoother, harder and in the laboratory have shown a reduced rate of volumetric wear (13). However there have been problems with ceramic THRs such as the recall of St. Gobain Desmarquest's ceramic femoral heads due to their high fracture rate after a manufacturing process change in 1998.

Acetabular wear results in changes in the shape and size of the volume occupied by the acetabular component. The terms linear wear and volumetric wear have been used in the literature to connote the largest depth of wear, $w$, and the total volume, $v$, of polyethylene lost, respectively (14). Modelling the worn volume as cylindrical gives $v=\pi r^{2} w$ where $r$ is the radius of the femoral head. Kabo et al. (15) found that the actual wear obtained from retrieval studies was only $53 \%$ of that estimated using this model. They proposed a refinement taking into account the direction of linear wear so that $v=\pi r^{2} w \frac{1+\sin (\beta)}{2}$ where $\beta$ is the angle between the acetabular rim and the direction of wear. In addition to wear, penetration can be caused by irreversible polyethylene deformation known as creep; polyethylene flows away from the area of high pressure and the femoral head penetrates the acetabular cup in the direction of the applied force. This does not cause loss of polyethylene and can in theory lead to overestimation of acetabular wear (16). However, polyethylene deformation has been found to be negligible after 12-18 months. Advances in the manufacture of polyethylene have all but eliminated deformation as a cause of prosthesis failure.

THRs are routinely assessed by x-ray imaging using plane radiographs ${ }^{1}$. During acquisition of an AP radiograph (Fig. 1), the radiologist aims the centre line of the radiation at the symphysis pubis. Film-focus distance is not always recorded but has been found experimentally to range from $900 \mathrm{~mm}$ to $1300 \mathrm{~mm}$ (18; 19) and is typically quoted as being

$\overline{1}$ CT is hampered by surface scatter from metal implants preventing it from being used to assess THR. Scatter correction has been proposed (17) but is not used in standard clinical practice. 


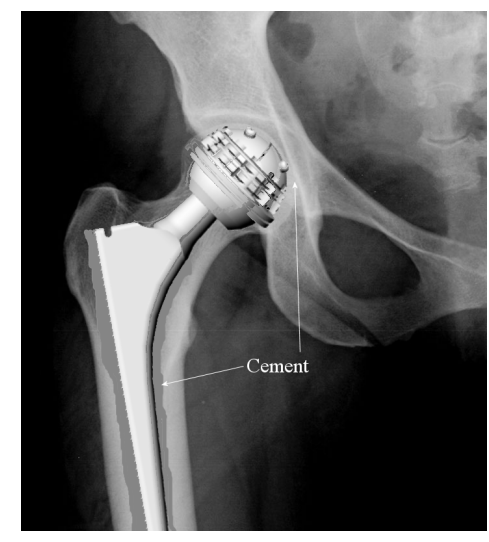

(a)

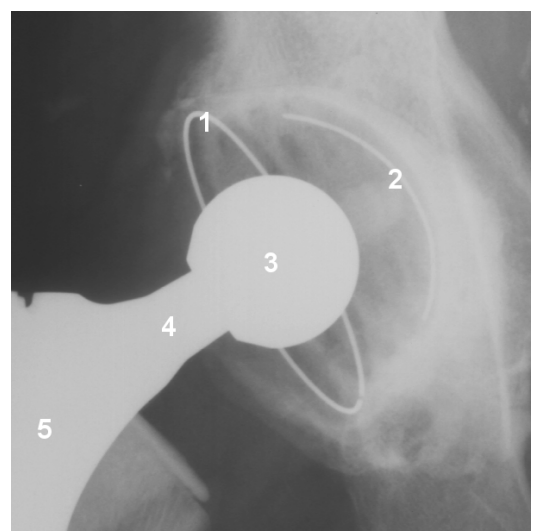

(b)

Fig. 1. (a) A diagram of a cemented prosthesis overlaid on an AP radiograph. (b) The region of interest on an AP radiograph showing (1) the acetabular rim wire marking the opening of the acetabular cup, (2) the edge marker used by Charnley to measure acetabular wear, (3) the femoral head, (4) the neck and (5) the stem of the prosthesis.

$1000 \mathrm{~mm}(20)$. The distance from the centre of the femoral head to the film has similarly been determined to range from $180 \mathrm{~mm}$ to $270 \mathrm{~mm}$. Some variation in rotation of the film plane relative to the beam centre (intrinsic rotation) and relative to the patient (extrinsic rotation) will also occur. Magnification can be corrected using the known diameter of the prosthetic femoral head. Mediolateral (ML) radiographs are more difficult to standardise than AP radiographs (20); contralateral structures can appear superimposed on the hip and consistent positioning of the patient can be problematic due to discomfort. Thus an ML radiograph is not always available to the clinician.

\section{Previous Work on Estimation of Acetabular Wear}

Existing methods for estimation of wear from radiographs can be categorised as uniradiographic, involving analysis of a single x-ray image, or duoradiographic, involving analysis of two radiographs taken at different times, typically the postoperative radiograph and the latest follow-up radiograph. It is difficult to make direct, quantitative comparisons between methods based on their reported accuracy or repeatability because of the varied ways in which these measurements are obtained and reported. Methods vary in the extent to which they are automated. Several aim to estimate linear wear solely within the AP view since this is where the majority of wear manifests itself (21). The earliest and still most widely used methods are manual in nature.

\subsection{Manual Methods}

Initial uniradiographic and duoradiographic studies conducted by Charnley et al. (22; 23) used a wire marker on the periphery of the acetabular cup (Fig. 1) and based wear estimates on two image distance measurements between the femoral head contour and the wire marker. These methods have since been criticised as unreliable, the uniradiographic 
method being more so, partly due to poorly controlled image acquisition (24). However, Griffith et al. (25) reported that the duoradiographic technique could give consistent wear measurements provided that the aim line was centered on the symphysis pubis and that the wire marker was not rotated more than $10^{\circ}$ out of the coronal plane. They estimated mean wear for a data set with an average age of 8.3 years as $0.07 \mathrm{~mm} /$ year, approximately half the rate reported previously by Charnley et al.. Wroblewski (26) compared estimates obtained using Charnley's duoradiographic method with measurments obtained in retrieval studies and found a strong correlation between them. They reported mean wear of $0.22 \mathrm{~mm} /$ year using Charnley's method against mean wear measured from retrieval of $0.19 \mathrm{~mm} /$ year.

Livermore et al. (14) proposed a duoradiographic method using a transparent overlay with a set of concentric circles at $1 \mathrm{~mm}$ increments of radius. The transparency was manually aligned on the radiograph film in order to estimate the centre of the femoral head. The shortest distance between the acetabular cup cement interface and the femoral head centre was estimated by means of a compass and caliper and estimates of the direction of wear were similarly obtained. Paired measurements of wear using Livermore's method were found by Martell et al. (27) to have a standard deviation of $0.72 \mathrm{~mm}$ when conducted by the same observer. Despite this poor repeatability, this method remains the most widely used for assessing acetabular wear (28). As Cowell (29) noted, there is considerable intra-personal and inter-personal variability when surgeons annotate a single line on a radiograph, never mind a method of this relative complexity.

\subsection{Interactive Computer-Assisted Methods}

Jones et al. (30; 31) first reported an interactive computer system (MAXIMA) to assist in localisation of prosthesis reference points such as the femoral head centre, stem midline and stem tip. Manually selected landmarks were used to initialise local contour searches based on custom edge enhancement filters. Active shape models have been applied to automatically and simultaneously segment contours of the femur, pelvis, stem and acetabular rim (32). They do not appear to have been applied to wear assessment.

Shaver et al. (33) created duoradiographic image analysis software for linear wear estimation with metal-backed acetabular cups. An initial estimate of the femoral head centre was based on a circle fitted to three user-defined points on the elliptical arc of the head contour. Radial lines were searched using an edge filter, resulting in a locus of points clustered around the contour of the femoral head. An ellipse was fitted to these points using a least-squares method (34). This procedure was then repeated to find the contour of the metal-backed acetabular cup. Wear estimates were based on changes in displacement of the femoral head centre relative to the acetabular cup centre and in separation of points on the elliptical arcs. When evaluated on radiographic data with ground-truth, a mean error of $3.6 \%$ was reported as compared to a mean error of $23.1 \%$ using the manual method of Livermore et al.

Chen et al. (35) fitted circles to femoral head and acetabular cup contours and used knowledge of the actual head and cup size along with imaging parameters to estimate 
the component of wear out of the plane of the radiograph. This duoradiographic method relied heavily on knowledge of imaging parameters such as the film-focus distance and did not model uncertainty in these parameters.

Similarly to Shaver et al. (33), the uniradiographic method of Hatfield et al. (36) began by fitting a circle to user-selected points on the femoral head contour. Radial searches were then performed for high gradients with appropriate intensity levels. The circular contour was then refined interactively through a process of manual outlier removal and automatic fitting. A similar approach was used to locate the acetabular cup edge wire albeit with a different filter and ellipse fitting. The removal of outliers was necessary especially in order to account for a radiopaque structure known as the mexican hat, a wire mesh used to plug gaps in the pelvis during surgery.

The uniradiographic method of Eggli et al. (37) also fitted a circle to manually selected points on the femoral head contour. However, it used the centre of the elliptical projection of the acetabular rim wire to estimate wear based on the distance in the image between it and the femoral head centre. The rim centre was computed based on manually selected points at the ends of the major axis of the elliptical rim contour. Annotation of the major axis was most likely very subjective. No account was taken of eccentricity error. The method also used the location of the teardrop feature of the pelvis to define a co-ordinate system for measuring wear.

Some methods can make use of the ML view when it is available in addition to the AP view in order to better estimate wear in 3D. It should be noted that these views are usually not acquired simultaneously, often require repositioning the patient, and are not calibrated with one another. Martell et al. (27) created a duoradiographic method for metal-backed prostheses. Two reference points were placed on the caudal most parts of the ischial tuberosities, an area of the lower part of the pelvis. Circles were fitted to the femoral head and acetabular cup using the Hough transform and ad hoc edge filters. The user could interactively constrain the fit by selecting regions of interest and annotating points on the contours. Knudsen et al. (38) reported that this method had a standard deviation of paired measurements of $0.332 \mathrm{~mm}$.

Devane et al. (39) attempted to measure 3D wear from AP and ML radiographs with metal-backed cups assuming that the beam centre and film-focus distance parameters were known. Circles were fitted to manually annotated points on the femoral head and acetabular cup contours in both views. Concentric ellipses were fitted to the inner and outer contours of the acetabular rim also based on manually annotated points. Visualisations of the cup and head were created using 3D models based on the manufacturer's specifications (Fig. 2). The accuracy of the method for estimating volumetric wear was reported as $\pm 0.411 \mathrm{~cm}^{3}$ based on an evaluation with 10 sets of AP and ML radiographs of cases with known volumetric wear. A version of this method is commercially available as Polyware and uses local feature search to refine the manually annotated points. However, it remains a highly interactive method and clinicians have reported finding the software interface "complex" (28).

None of the methods described above for measuring acetabular wear are fully automatic. Human annotation thus introduces intra-observer and inter-observer variability. Several 


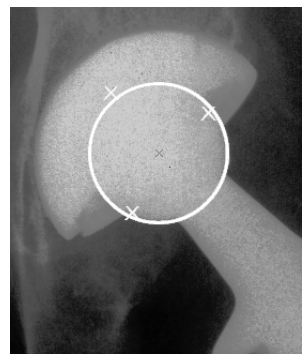

(a)

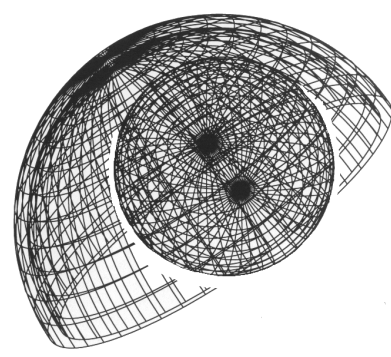

(b)

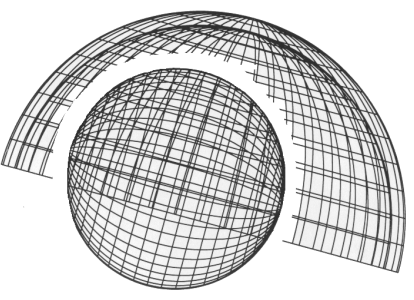

Fig. 2. (a) Circle fitting and (b) visualisations of AP and ML views created using Polyware.

of the methods assume that the cup is metal-backed. The remainder of this paper is concerned with automating analysis of prostheses with acetabular cups that are not metal backed. The image of the wire marker at the rim of the acetabular cup will be used for this analysis.

\section{Modelling femoral head and acetabular rim contours}

The acetabular rim wire marker and the articular surface of the femoral head can be modelled as circular and spherical, respectively. The femoral head gives rise to an elliptical arc in the radiograph which is extremely close to circular. The acetabular rim marker, conversely, projects as a clearly non-circular ellipse due to (i) rotation in depth and (ii) translation away from the x-ray beam centre. Localising the femoral head and acetabular rim can therefore be formulated as the problem of localising two elliptical structures in the AP image. The elliptical projections of acetabular rims in standard clinical radiographs typically have eccentricities 2 between 0.8 and 1.0. When, in extreme cases, the circular contour of the acetabular rim projects as a linear structure (Fig. 3), the method cannot be used to localise the contour.

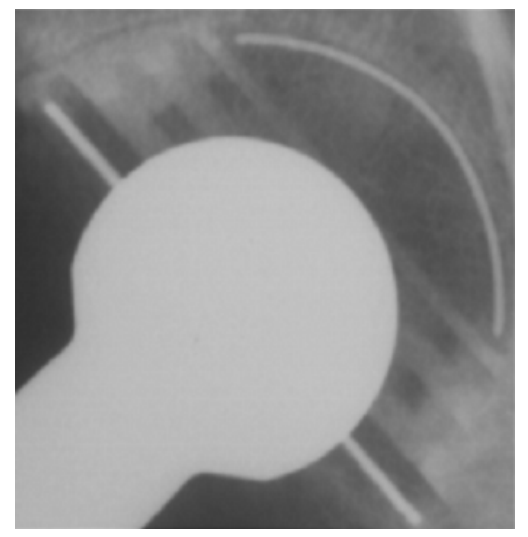

Fig. 3. Extreme rotation in depth can result in the acetabular rim marker projecting as a linear structure.

Previous work on radiographic THR analysis has used general purpose or ad hoc edge

2 Eccentricity is defined as $e=\sqrt{1-\frac{b^{2}}{a^{2}}}$, where $a$ and $b$ are the major and minor semi-axes lengths. 
filters to model the local appearance of structures of interest (30; 33, 36). In preference to such an approach, local appearance models are learned here from annotated training images, similarly to the approach commonly adopted with active shape models (40). A training set is prepared by manually annotating points on contours in training examples. Specifically, points at the start and end of the elliptical arc of the femoral head contour were annotated. Further points were then anotated by repeatedly selecting points midway, in terms of arclength, between existing points. A second set of points was annotated on the acetabular rim wire with points more closely spaced at higher curvature. Ellipses were automatically fitted to these point sets using least-squares fitting. Fig. 4 shows an example.

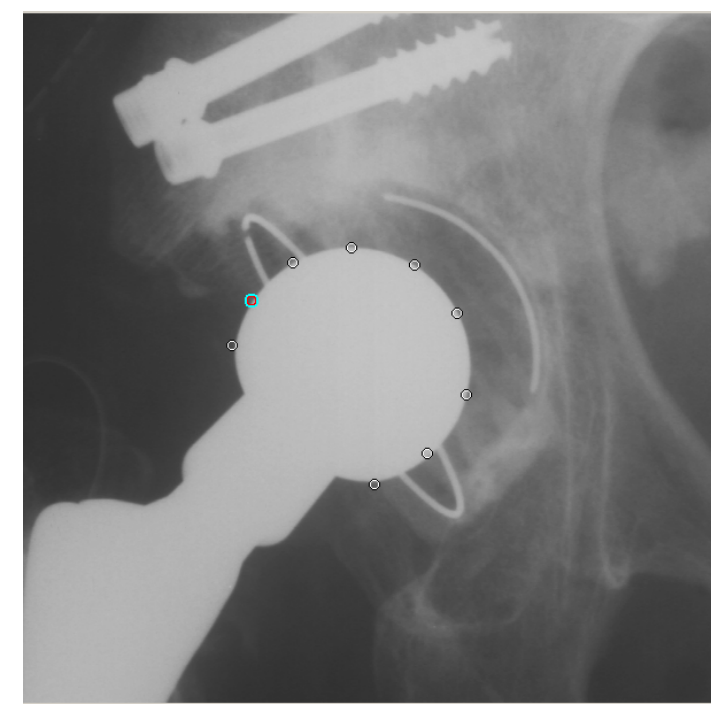

(a)

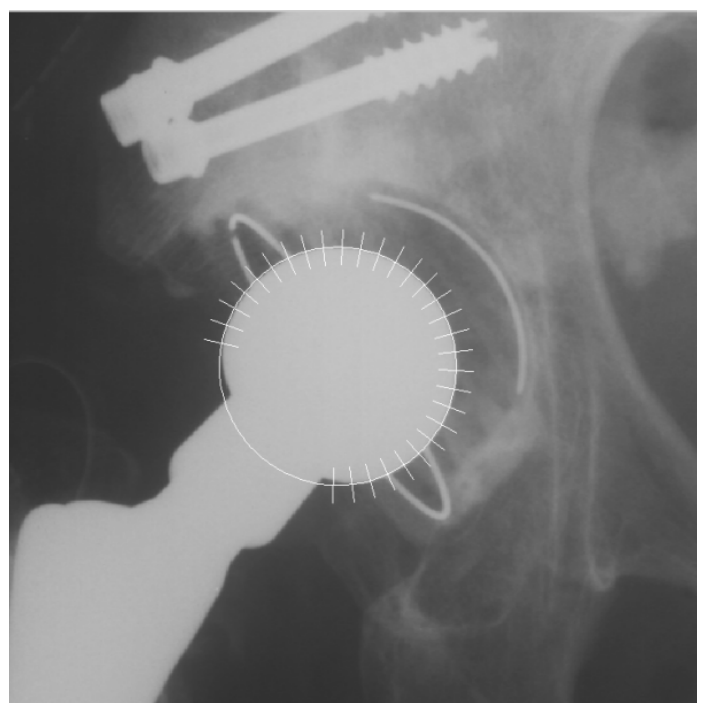

(c)

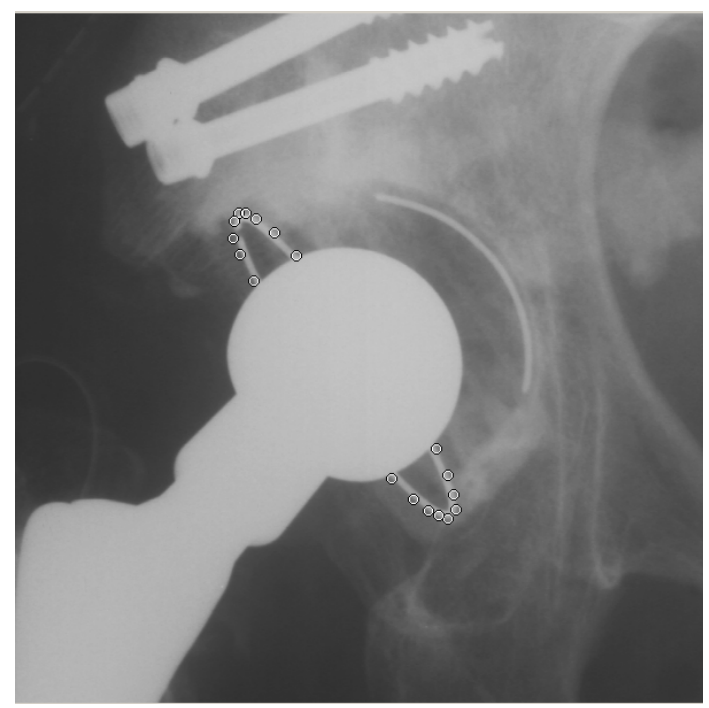

(b)

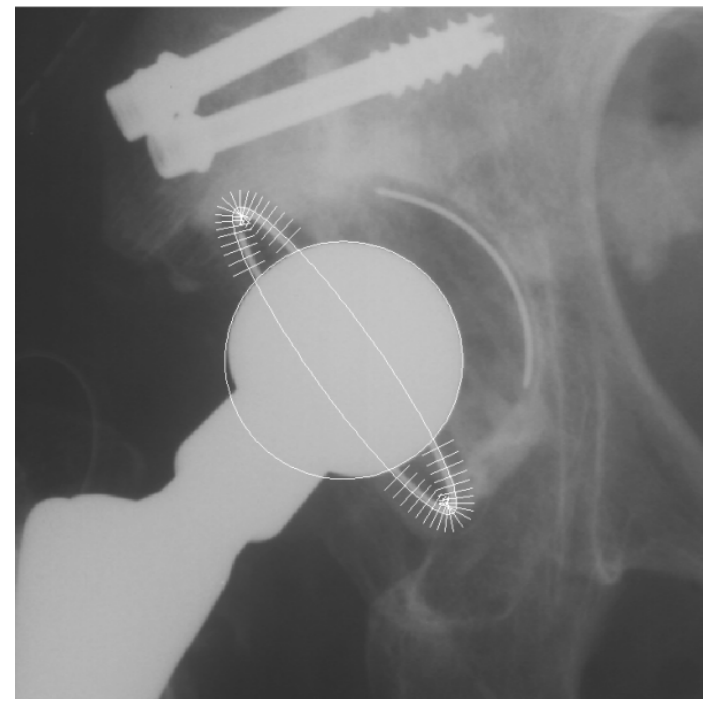

(d)

Fig. 4. (a,b) Annotated points on the femoral head and acetabular rim of a training example. (c,d) Ellipses fitted to these point sets (with ellipse normals also shown).

Equation (1) gives a conic section when $E_{a}(x, y)=0$ and an ellipse when $B^{2}-4 A C<0$.

$$
E_{a}(x, y)=A x^{2}+B x y+C y^{2}+D x+E y+F
$$


Least squares ellipse fitting involves minimising $\sum_{i}\left(E\left(x_{i}, y_{i}\right)\right)^{2}$ where the sum is over the data points and $E\left(x_{i}, y_{i}\right)$ is a suitable error function evaluated at the $i^{\text {th }}$ point. The algebraic error function (Equation 1) is commonly used and closed form solutions incorporating the ellipse constraint are available $(34 ; 41)$. However, algebraic fitting has limitations, particularly when fitting eccentric ellipses such as those of the acetabular rim. Firstly the algrebraic error is not invariant under Euclidean transformation. Secondly, it exhibits a high degree of curvature bias, meaning that a point at a high curvature portion of the contour contributes less to the fitting than a point having the same amount of noise but at a lower curvature portion of a contour, leading to overly eccentric or shrunk ellipses. Thirdly, it weights points on the exterior of the ellipse more strongly than those in the interior.

The most obvious alternative choice of error function is the orthogonal distance, i.e. the geometric distance from a point to its closest point on the ellipse curve, known as the orthogonal contacting point. However, this distance is not trivial to compute and thus numerous computationally cheaper error functions have been considered in the past (42, 43). Today's computational power and the availability of an efficient algorithm (44; 45) have increased the feasibility of using the orthogonal distance. The orthogonal contacting point can be found using Gauss-Newton minimisation.

The fitting strategy adopted here was to first perform a least squares algebraic (LSA) fit using the algorithm of Halir and Flusser (41) and to subsequently refine it using a least squares orthogonal distance (LSO) fit (44) (step size $\kappa=1.2$ ). The underlying assumption in adopting an LSO fit is that annotation displacement errors normal to the ellipse contour are Gaussian distributed. LSA fitting on its own was found to hamper fits to the acetabular rim as the curvature bias perturbed fits to noisy and incomplete datasets. An example of LSA producing a wildly different fit from LSO is shown in Fig. 5.

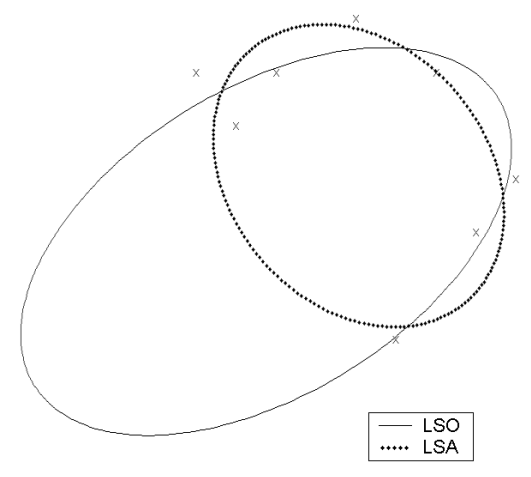

Fig. 5. Comparison of fits to the same data points using LSO (initialised with a circle fit) and LSA.

Once ellipse fitting was completed, grey-level derivative profiles were sampled along normals centred on each of the elliptical contours. Derivative profiles were estimated as finite difference approximations and normalised so that their elements summed to unity. In the case of the femoral head it was necessary to discount normals on the neck of the prosthesis. In the case of acetabular rim ellipses, normals that passed through the femoral head ellipse were discarded. Sample means and covariances matrices were computed from the remaining normalised profiles for the femoral head and for the acetabular rim respectively. 
Fig. 6 shows the sample means along with standard deviations.
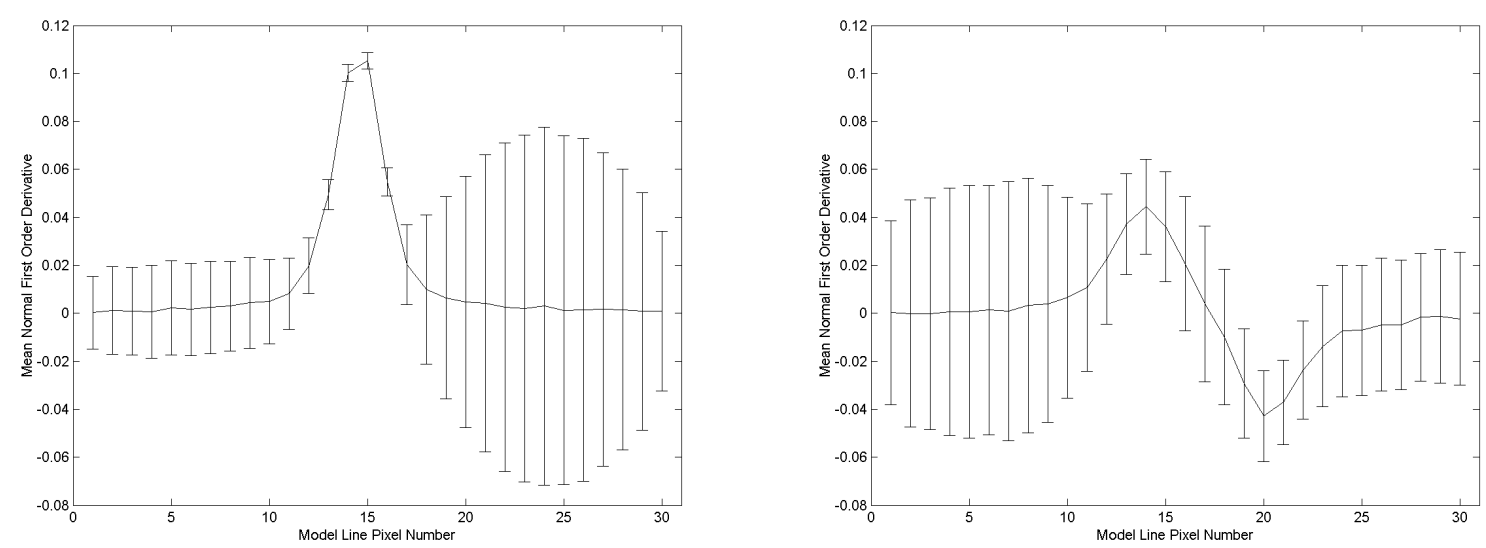

Fig. 6. Plots of the mean profiles, with error bars showing one standard deviation, obtained from 50 postoperative radiographs for the femoral head (left) and the acetabular rim (right)

\section{Active Ellipse Search}

A simple, reduced resolution template matching was effective in providing a coarse localisation of the femoral head in order to initialise local search. Specifically, the maximum of the normalised cross-correlation with a template was found where the template consisted of a cropped femoral head image. When evaluated on $600 \times 600$ pixel cropped regions of interest from 100 radiographs of Zimmer CPT protheses scanned at 150 dpi, this straightforward method resulted in successful localisation of the femoral head in all cases. However, localisation of the femoral head in uncropped radiographs would require a more advanced method of initialisation. This is not pursued further here.

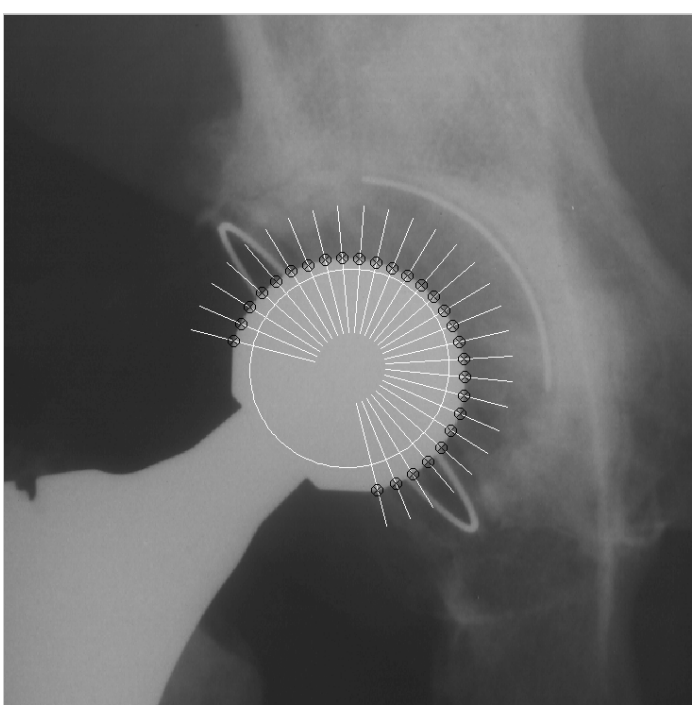

(a)

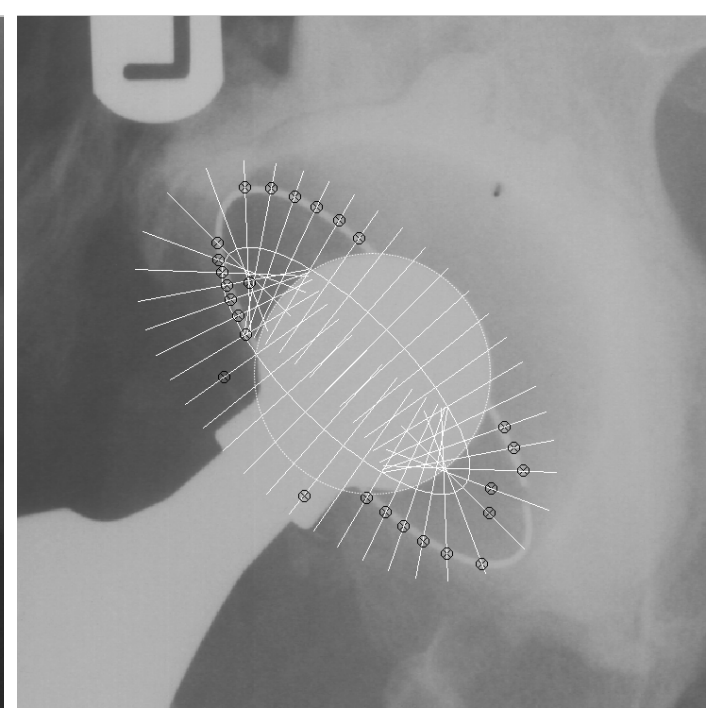

(b)

Fig. 7. Points on (a) the femoral head and (b) the acetabular rim, found by minimising Mahalanobis distances over search normals of length 111 and 151 pixels respectively. 


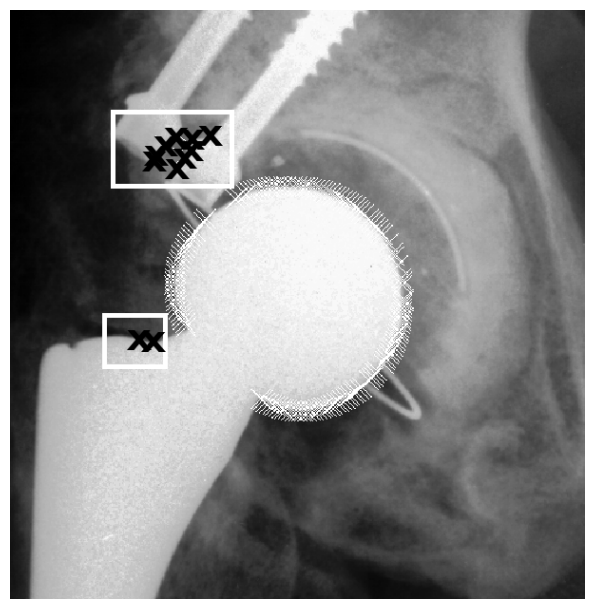

(a)

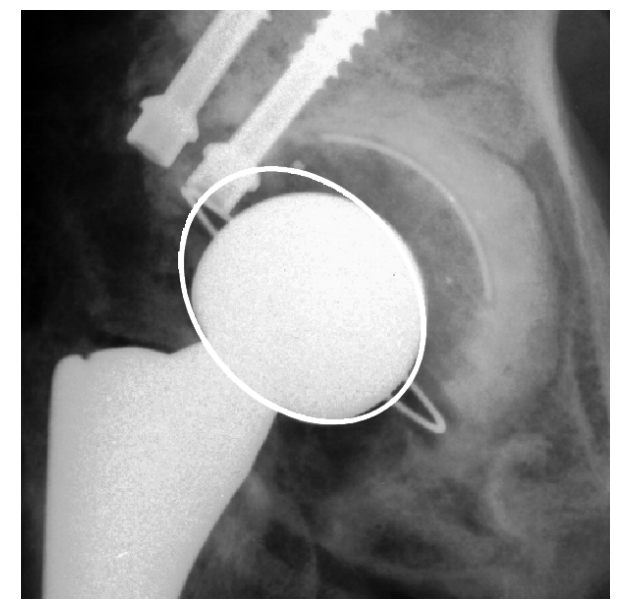

(b)

Fig. 8. (a) Points found during femoral head search many of which do not correspond to the elliptical head contour. (b) A least-squares ellipse fit to these points.

Initial estimates of an ellipse's parameters were refined by iterative local search in the manner of active shape model search. At each iteration, search line segments were centred on the current contour normal to it. The normalised derivative profile on each search line with minimum Mahalanobis distance to the model was found. Minimising the Mahalanobis distance maximised the probability that the observed profile was drawn from the assumed Gaussian distribution. This resulted in a new set of points, one on each search line. The ellipse parameters were updated by fitting to these new points. This was iterated to convergence or until a fixed maximal number of iterations was completed. A check was then made for grossly implausible parameters as an indication of fitting failure. In such cases, active ellipse search was reinitialised. The process is illustrated in Fig. 7.

Femoral head search was performed first. Once the head was localised, acetabular rim search was initialised with the acetabular ellipse centre coincident with the femoral head centre and its other parameters initialised as sample means from the training set. In the case of the femoral head it was necessary to automatically discount datapoints in the neck region. Up to approximately one-quarter of the ellipse can lie in the neck. Therefore, the derivatives were summed for each matching profile and the $25 \%$ with the lowest sums were discarded. In the case of the acetabular rim, points were discarded that were within the femoral head.

\section{Robust Fitting}

Individual points found during active ellipse search are often not on the structure of interest. Fig. 8(a) shows an example in which outliers are prevalent due to radiopaque clutter, in this case a pair of screws to aid fixation of the acetabular cup, the neck of the prosthesis and confusion between the head and rim. The points in the neck can be discarded as described above. Clearly, the distribution of the remaining points around the contour is not well modelled as zero-mean Gaussian. Least-squares ellipse fitting can therefore result in a very poor fit (Fig. 8(b)). LS has a breakdown point of $0 \%$ outliers, 
so even a single outlier can result in dramatic failure. Instead, a robust fitting method capable of tolerating high numbers of outliers is required (46).

Least median of squares (LMedS) fitting, in which $\operatorname{med}_{i}\left(E\left(x_{i}, y_{i}\right)\right)^{2}$ is minimised, has been found to give good results for ellipse fitting in the presence of outliers (47; 42; 48) and has also been applied to active shape model search (49, 50). It has a breakdown point of $50 \%$. In LMedS ellipse fitting, 5-point subsets are randomly selected and the residuals for the complete data set are computed for those that define ellipses. The ellipse that minimises the median error is selected. This fit can be "polished" by performing a least-squares fit to the set of inliers as determined from the LMedS fitted ellipse. Inliers can be defined by placing a threshold on the point errors, so that the $i^{\text {th }}$ point is selected for LS fitting if and only if it satisfies $\left|E\left(x_{i}, y_{i}\right)\right|<3 \gamma d$ where $d$ is the median absolute deviation (a robust noise estimate) defined as:

$$
d=\operatorname{med}_{i}\left|\left(E\left(x_{i}, y_{i}\right)\right)^{2}-\operatorname{med}_{j}\left(E\left(x_{j}, y_{j}\right)\right)^{2}\right|
$$

and $\gamma$ is a Gaussian normalisation and finite-sample correction factor (51):

$$
\gamma=1.4826\left(1+\frac{5}{n-1}\right)
$$

The number, $h$, of 5 -point subsets to evaluate is typically determined in advance. If the fraction of data points that are outliers is $\epsilon$, the probability of selecting 5 points that are all inliers is given by

$$
P=1-\left(1-(1-\epsilon)^{5}\right)^{h}
$$

and rearranging gives an expression for $h$ :

$$
h=\frac{\log (1-P)}{\log \left(1-(1-\epsilon)^{5}\right)}
$$

However, Equation (5) does not take into account the quality of the inliers. Given, noisy inlying points, it is advisable to use a larger value of $h$.

As noted earlier, several error of fit functions have been used for ellipse fitting and these vary in terms of computational cost, curvature bias and asymmetry bias, for example (43, 52, 53). The algebraic distance and the weighted algebraic distance are commonly used. Rosin (43; 52) compared 13 error functions and found that the foci bisector distance provided the best goodness of fit, the lowest curvature bias and an acceptable level of asymmetry. In this paper, an orthogonal distance error function is compared to the algebraic, weighted algebraic and foci bisector errors. There appears to be no previous work using the orthogonal distance as an error function for LMedS ellipse fitting. The experiments reported in what follows are also unusual in that the acetabular rim ellipses are more eccentric than those found elsewhere in the ellipse fitting literature. 
The algebraic error function, $E_{a}$, was given in Equation (1). The weighted algebraic distance, $E_{w a}$, is the algebraic distance inversely weighted by its gradient:

$$
E_{w a}(x, y)=\left(A x^{2}+B x y+C y^{2}+D x+E y+F\right) \frac{2 C y_{i}+B x_{i}+E}{2 A x_{i}+B y_{i}+D}
$$

This can exhibit a lower curvature bias but a greater asymmetry than $E_{a}$; points within the ellipse are weighted more heavily than points outside the ellipse. The foci bisector distance is illustrated in Fig. 9 and is defined as

$$
E_{f b}(x, y)=\sqrt{\left(x_{f}-x\right)^{2}+\left(y_{f}-y\right)^{2}}
$$

where $\left(x_{f}, y_{f}\right)$ is the closest point of intersection of the ellipse with the angular bisector of the lines through the point and the ellipse foci. The foci are given by the expression

$$
\left(x_{c}, y_{c}\right) \pm\left(\sqrt{\left(a^{2}-b^{2}\right)} \cos \theta, \sqrt{\left(a^{2}-b^{2}\right)} \sin \theta\right)
$$

where $\left(x_{c}, y_{c}\right)$ is the ellipse centre, $a$ is the major semi-axis length, $b$ is the minor semi-axis length and $\theta$ its orientation.

The orthogonal distance, $E_{o}$, between a point and an ellipse is the minimum Euclidean distance between the point and that ellipse:

$$
E_{o}(x, y)=\sqrt{\left(x-x_{o}\right)^{2}+\left(y-y_{o}\right)^{2}}
$$

where $\left(x_{o}, y_{o}\right)$ is the orthogonal contacting point. Both Safaee-Rad et al. (53) and Ahn et al. (44; 45) proposed methods for locating the orthogonal contacting point. Without loss of generality, consider a canonical ellipse centred at the origin and with its axes aligned with the image axes.

$$
\frac{x^{2}}{a^{2}}+\frac{y^{2}}{b^{2}}=1
$$

An orthogonal contacting point will satisfy:

$$
\frac{\delta y}{\delta x} \cdot \frac{y_{i}-y}{x_{i}-x}=\frac{-b^{2} x}{a^{2} y} \cdot \frac{y_{i}-y}{x_{i}-x}=-1
$$

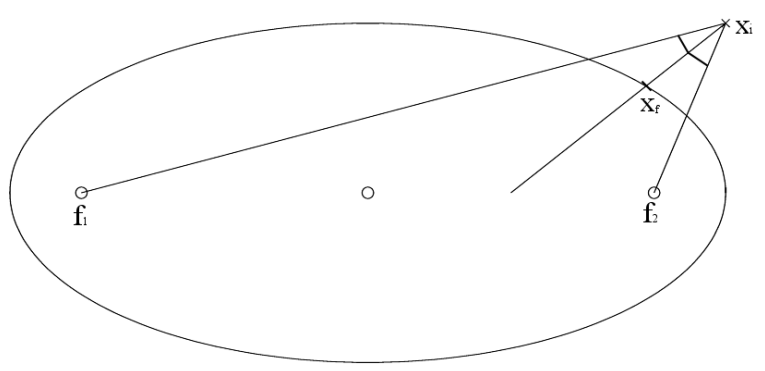

Fig. 9. The closest point of intersection of the angular bisector $\left(\mathbf{x}_{f}\right)$ 
Combining Equations (9) and (10) gives:

$$
\begin{aligned}
& f_{1}(x, y)=\frac{1}{2}\left(a^{2} y^{2}+b^{2} x^{2}-a^{2} b^{2}\right)=0 \\
& f_{2}(x, y)=b^{2} x\left(y_{i}-y\right)-a^{2} y\left(x_{i}-x\right)=0
\end{aligned}
$$

A solution was found using the generalized Newton method. This iterative procedure was initialised with the intersection point on the ellipse of a line from the centre of the ellipse and the data point. This is a good initialisation as it always lies in the same quadrant of the ellipse as the orthogonal contacting point. Ater 3 to 4 iterations, convergence was reached upon the orthogonal contacting point.

\subsection{Empirical Comparison of Ellipse Fitting Methods}

\subsubsection{Synthetic Data}

LMedS ellipse fitting using each of the four error functions $E_{a}, E_{w a}, E_{f b}$ and $E_{o}$ was tested for accuracy and robustness using synthetic data sets somewhat similar to those used by Rosin (42). Two ellipses were used to generate them; the first had an eccentricity of 0.66 $(a=333, b=250)$ and the second had an eccentricity $0.98(a=300, b=60)$. Three types of data set were generated using each ellipse.

Gaussian noise Data points were sampled at uniform intervals along the ellipse with additive Gaussian noise in the direction normal to the ellipse contour $(\sigma=0,10, \ldots, 70)$.

Gaussian outliers Some points were sampled with noise drawn from a low variance Gaussian $(\sigma=5)$ while the remainder had high variance Gaussian noise $(\sigma=500)$ thus creating outlying points. The percentage of points with high variance noise was varied $(0 \%, 10 \%, \ldots, 90 \%)$.

Structured outliers Structured noise was introduced by sampling some of the points from straight line segments close to the ellipse. All points were sampled with Gaussian noise $(\sigma=5), 80 \%$ from an elliptical arc, $10 \%$ from a line segment orthogonal to that arc, and $10 \%$ from another line segment rotated $45^{\circ}$ with respect to the first line segment. The percentage of structured outliers was varied $(0 \%, 10 \%, \ldots, 90 \%)$.

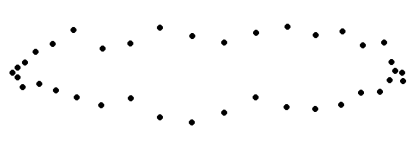

(a)

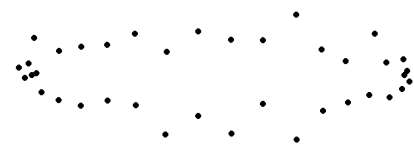

(b)

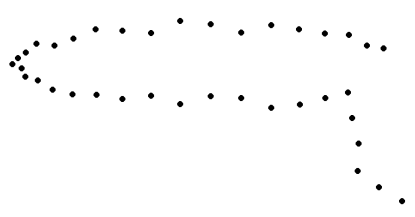

(c)

Fig. 10. Point sets generated from the more eccentric ellipse using (a) Gaussian noise $(\sigma=10)$, (b) half low-variance Gaussian noise $(\sigma=5)$ and half high-variance Gaussian noise $(\sigma=20)$, and (c) structured outliers sampled from noisy line segments.

An example from each type of data set is shown in Figure 10. Each example consisted of 38 points. LS fits using orthogonal and algebraic error functions and LMedS fits (with LS fine 
tuning on resulting inliers) using algebraic, weighted algebraic by gradient, foci bisector distance and orthogonal error (44) functions were performed. The Euclidean distances between the original and recovered ellipse centres were used as a measure of accuracy.
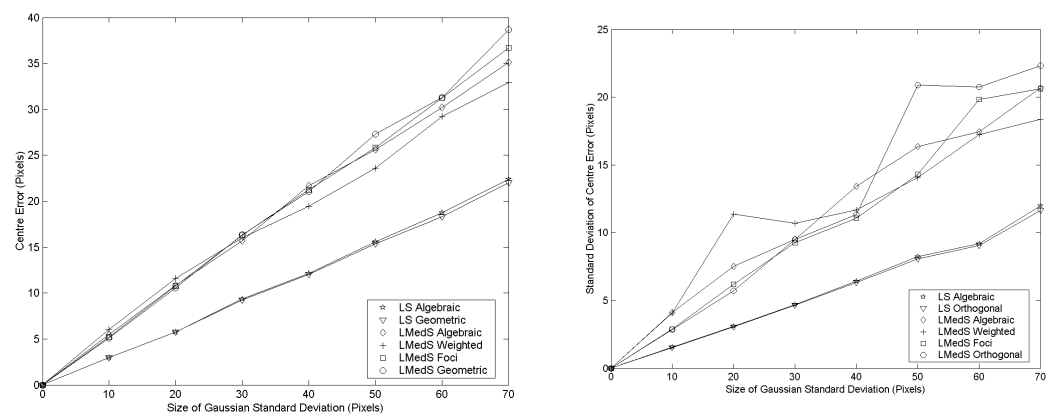

(a)
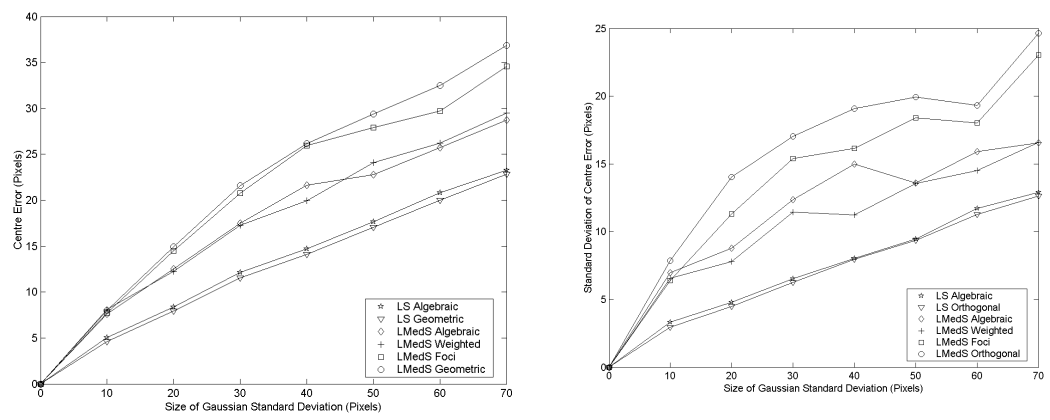

(b)

Fig. 11. Centre errors (means and standard deviations) for (a) less eccentric and (b) more eccentric synthetic ellipse data with Gaussian noise

Figs. 11-13 show the alpha trimmed means $(\alpha=0.1)$ of the centre errors. Each point on these plots was computed from 500 examples. Also shown are the standard deviations of the 500 centre errors obtained per dataset.

Unsurprisingly, LS outperformed LMedS in the presence of purely Gaussian noise (see Fig. 11) with orthogonal LS only slightly more accurate than algebraic LS. However, the difference between orthogonal and algebraic LS is more pronounced on the more eccentric ellipse.

LMedS fitting was more accurate in the presence of outliers, whether Gaussian or structured. Figs. 12 and 13 show that LMedS using foci bisector or orthogonal error functions performed best on both ellipse eccentricities. It can be seen from Fig. 14 that the orthogonal distance outperforms the foci bisector slightly. Results obtained with greater than $50 \%$ outliers lie beyond the theoretical break-down point of LMedS and so not surprisingly are poor. LMedS fitting with algebraic and or weighted algebraic functions performed very poorly in the presence of structured noise (Fig. 13). In fact, LS methods were better in this case as LS fitted to both the straight line segments and the elliptical arc, evidenced by the relatively low standard deviations of both LS fits. The LMedS algebraic and weighted algebraic tended to favour points on the line segments and thus had very high standard deviation. 

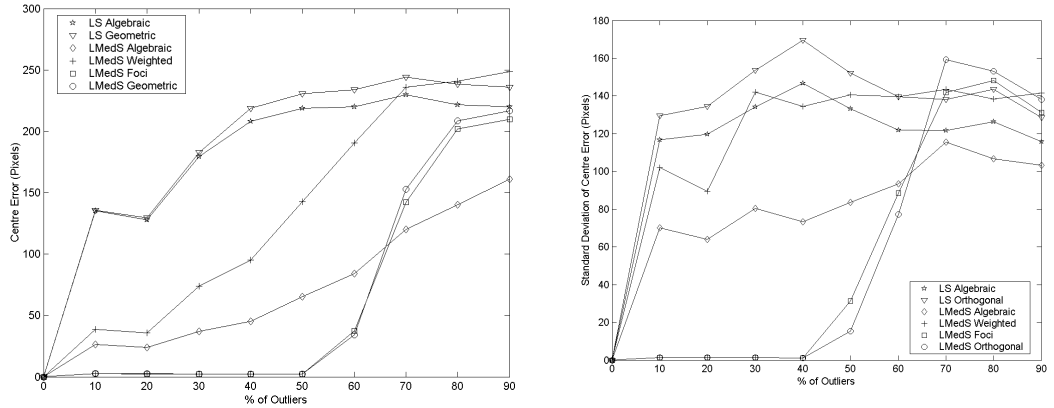

(a)
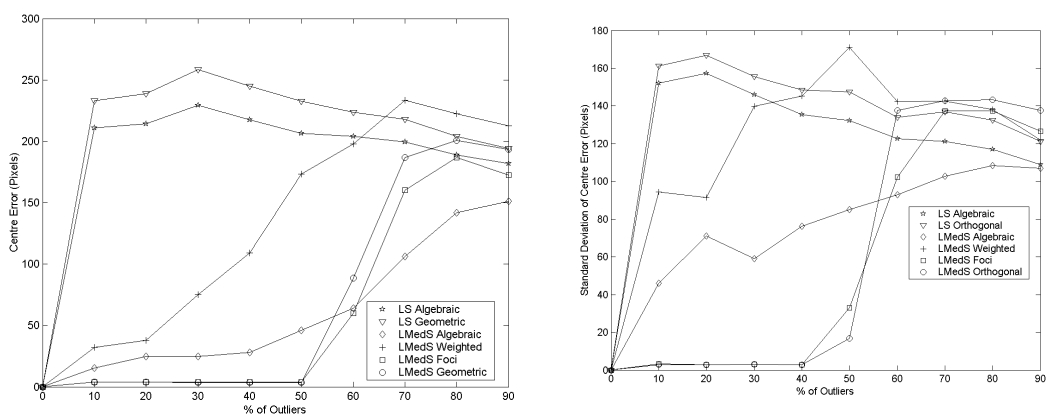

(b)

Fig. 12. Centre errors (means and standard deviations) for (a) less eccentric and (b) more eccentric synthetic ellipse data with Gaussian outliers
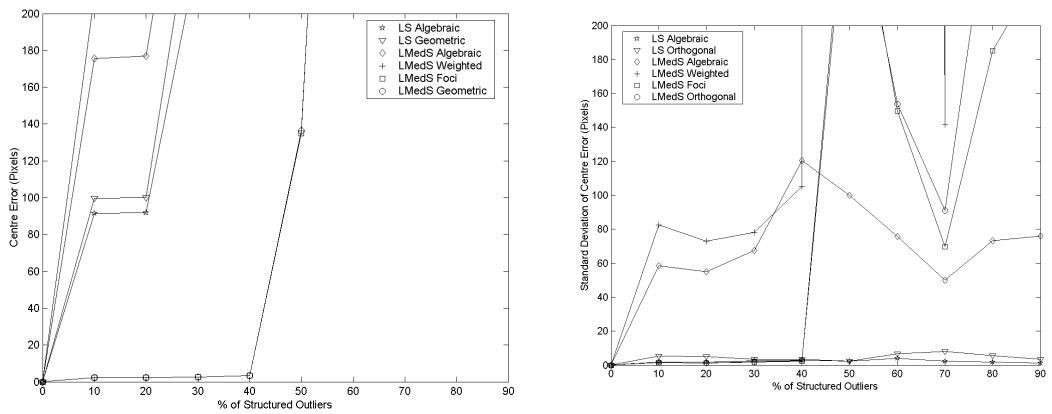

(a)
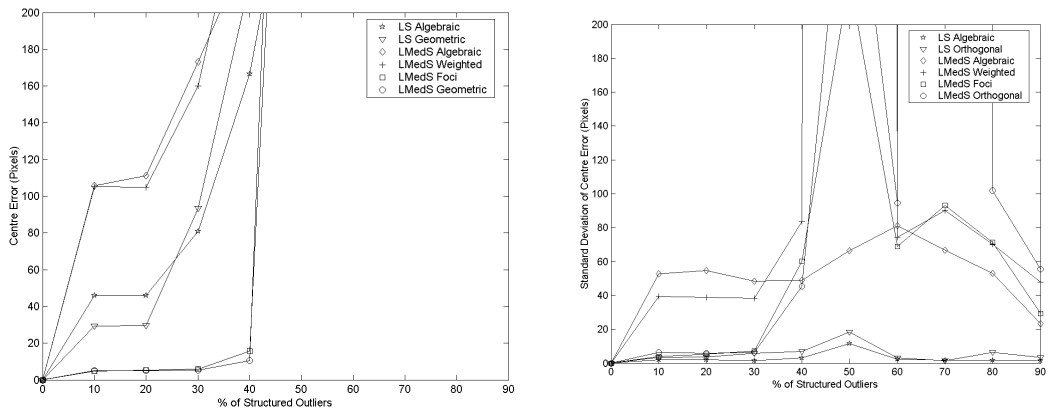

(b)

Fig. 13. Centre errors (means and standard deviations) for (a) less eccentric and (b) more eccentric synthetic ellipse data with structured outliers 


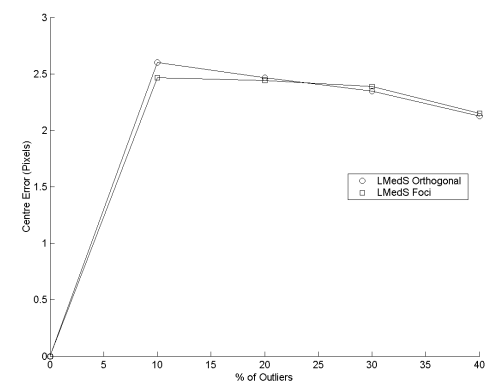

(a)

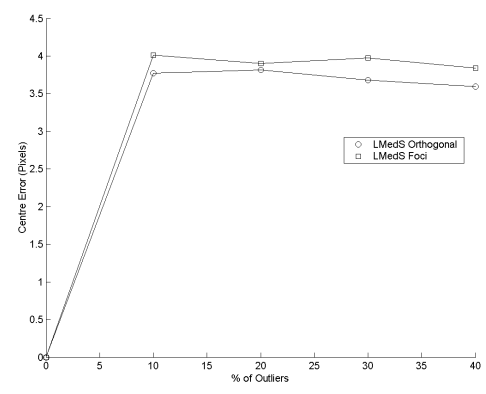

(c)

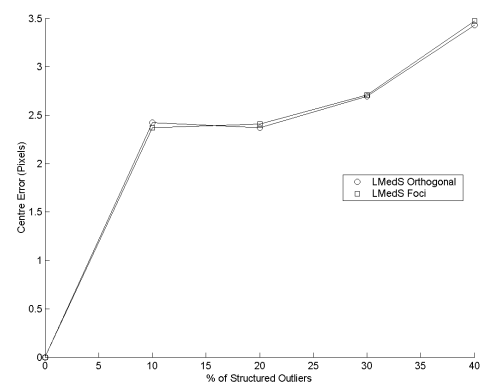

(b)

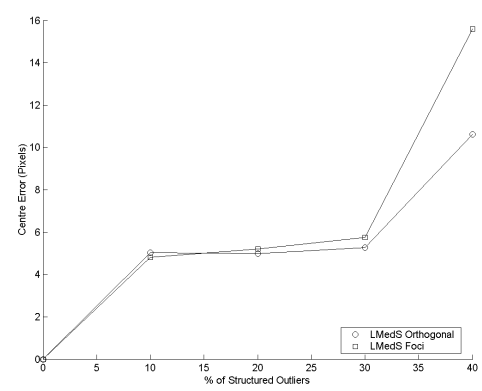

(d)

Fig. 14. Centre errors using foci bisector and orthogonal distance error functions for the less eccentric ellipse with (a) Gaussian outliers and (b) structured outliers, and for the more eccentric ellipse with (c) Gaussian outliers and (d) structured outliers.

\subsubsection{Radiographic Data}

A set of 19 radiographs containing Zimmer CPT prostheses with particularly eccentric rim projections $(e>0.96)$ was obtained. The most accurate error-of-fit functions from the experiments using the synthetic data sets described above were selected. These were used to perform active ellipse localisation on the radiographs. Localisation was run twice on each image, providing 38 results per method. No LS fine tuning was performed on the resulting inliers.

On this challenging dataset, LMedS orthogonal fitting succeeded 23 times out of 38, LMedS foci bisector 22 times, and LMedS algebraic 6 times. An example of the output of each of these algorithms is shown in Figure 16. Fig. 15 shows examples of failures. Given the excessive difficulty of the data set and the absence of any LS fine tuning to inliers (which increases the performance of all three error functions), the results using LMedS fitting were encouraging considering typical datasets include eccentricity ranging from 0.8 to 1.0 while this dataset focused on eccentricities higher than 0.96 . The orthogonal distance was retained as an error function in the experiments that follow in order to obtain high accuracy. 


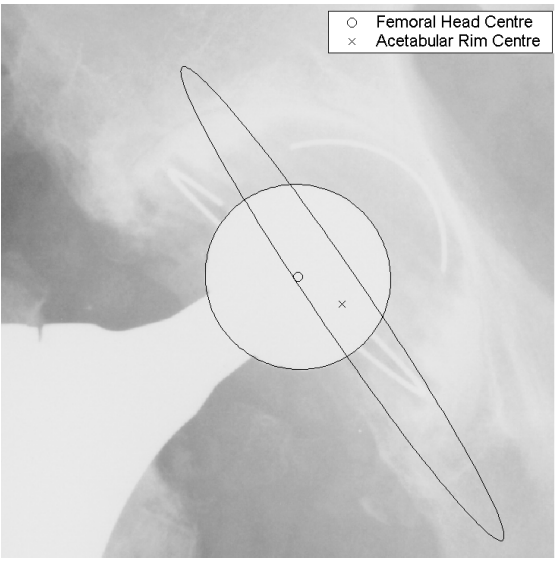

(a)

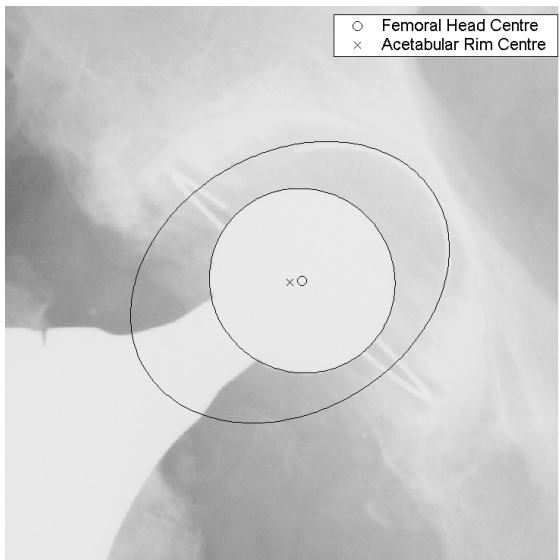

(c)

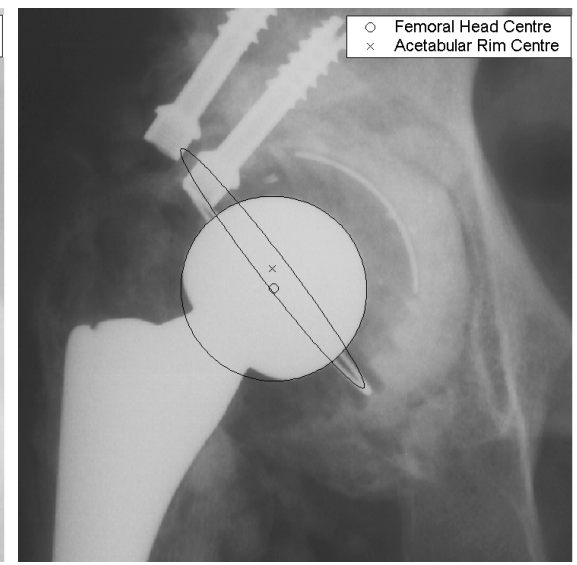

(b)

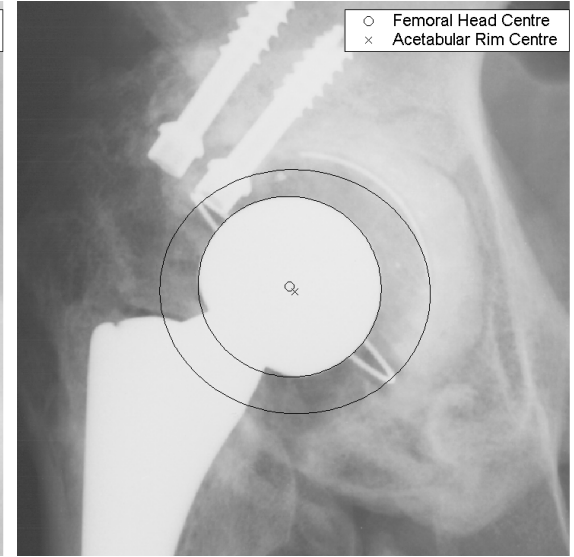

(d)

Fig. 15. Failures of the method due to (a) a highly eccentric rim at an unusual orientation, (b) clutter, and (c-d) fitting to the near-circular wire marker on the periphery of the acetabular cup.

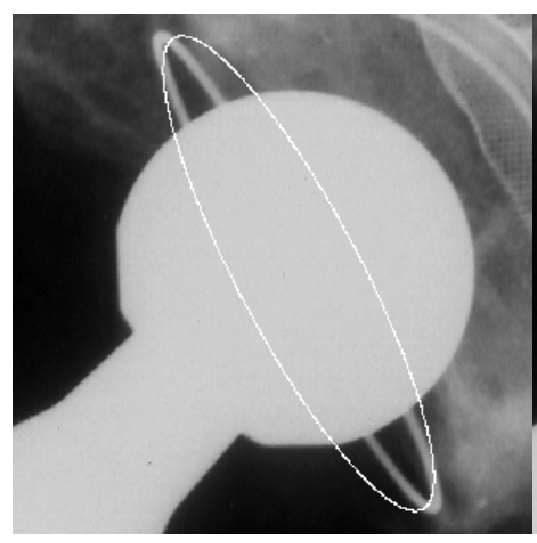

(a)

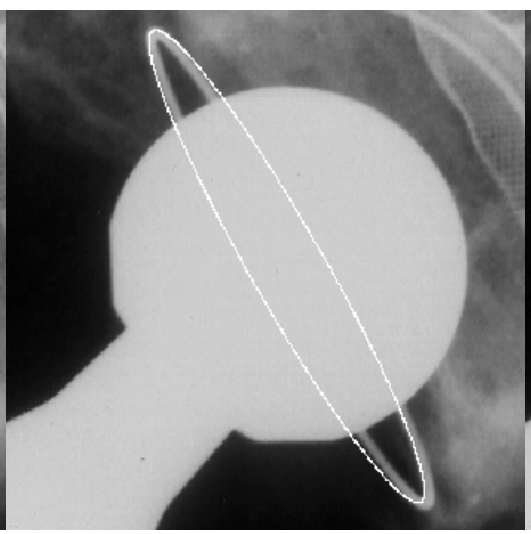

(b)

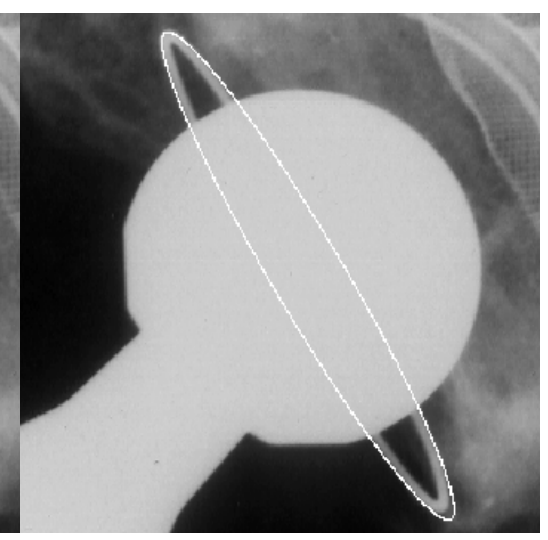

(c)

Fig. 16. An eccentric rim with (a) failed algebraic, (b) successful foci bisector and (c) successful geometric fits. 


\section{Wear Estimation}

Acetabular wear manifests as displacement over time of the acetabular rim relative to the femoral head. Given the image projections of the 3D centres of the acetabular cup rim and the femoral head, wear in the plane of radiograph could be measured in terms of their relative displacement based on the known diameter of the femoral head. Specifically, let $\mathbf{x}_{c}^{(t)}=\left(x_{c}^{(t)}, y_{c}^{(t)}\right)$ and $\mathbf{x}_{c}^{(t+\Delta t)}=\left(x_{c}^{(t+\Delta t)}, y_{c}^{(t+\Delta t)}\right)$ denote the image coordinates of the projections of the cup rim centre in radiographs acquired at times $t$ and $t+\Delta t$. Similarly, let $\mathbf{x}_{h}^{(t)}$ and $\mathbf{x}_{h}^{(t+\Delta t)}$ denote images of the femoral head centre. Linear wear, $w(t, \Delta t)$, between times $t$ and $\Delta t$ could be approximated as:

$$
w(t, \Delta t)=\rho^{(t+\Delta t)}\left(\mathbf{x}_{r}^{(t+\Delta t)}-\mathbf{x}_{h}^{(t+\Delta t)}\right)-\rho^{(t)}\left(\mathbf{x}_{r}^{(t)}-\mathbf{x}_{h}^{(t)}\right)
$$

where $\rho^{(t)}$ and $\rho^{(t+\Delta t)}$ are factors that convert the units of measurement from pixels to $m m$. Let $a_{h}^{(t)}$ and $b_{h}^{(t)}$ denote the major and minor semi-axis lengths of the femoral head ellipse. Given the radius, $r$, of the femoral head, the conversion factor at time $t$ is:

$$
\rho^{(t)}=\frac{2 r}{a_{h}^{(t)}+b_{h}^{(t)}}
$$

and similarly for $\rho^{t+\Delta t}$. It is important to note, however, that the centre of the acetabular rim ellipse does not in general coincide with the image projection of the centre of the rim in 3D nor consistently to any point on the prosthetic structure. Nevertheless, the ellipse centre has been suggested as a reference point for measuring acetabular wear (19; 37). The discrepancy between the centre of the projected ellipse and the projection of the centre of the circle is known as the eccentricity error and is illustrated in Fig. 17. Eccentricity error is largely due to rotation in depth so it is useful to be able to estimate the rotation of the circular rim marker relative to the image.

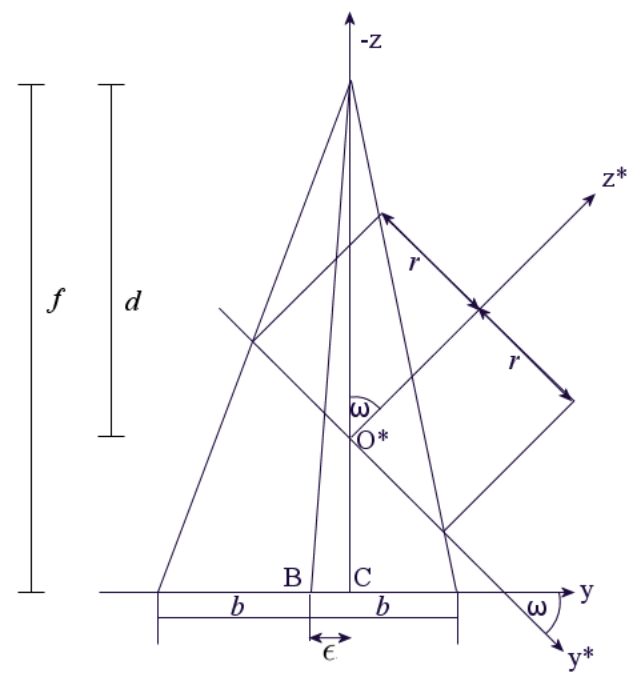

Fig. 17. Projection of the circle to an ellipse showing the ellipse's minor axis. Eccentricity error, $\epsilon$, is the distance between the ellipse centre, $B$, and the projection, $C$, of the circle's centre, $O^{*}$. 


\subsection{Estimation of Rotation in Depth and Eccentricity Error}

The 3D pose of the acetabular rim circle can be estimated from its image provided that the film-focus distance, $f$, is known. Consider the cone of perspective projection that intersects the acetabular rim ellipse and the centre of projection. The pose can be found as the rotation such that the intersection of this cone with the image plane becomes a circle. Forsyth et al. (54) suggested computing this rotation in two stages. The first stage computes a rotation which centres the projected ellipse at the image centre with its major axis aligned with the image $x$-axis. This is achieved by diagonalising the ellipse matrix. The second stage computes a rotation about the $x$-axis in order to obtain a circle. More formally, if all distances are expressed as multiples of $f$, the equation of the cone (formed by the ellipse and the centre of projection) in matrix form is $\mathbf{x}^{T} \mathbf{E x}=0$, where $\mathbf{x}=[x, y, z]^{T}$ and $\mathbf{E}$ is the real, symmetric matrix

$$
\mathbf{E}=\left[\begin{array}{ccc}
A & B / 2 & D / 2 \\
B / 2 & C & E / 2 \\
D / 2 & E / 2 & F
\end{array}\right]
$$

The first rotation, $\mathbf{R}$, of this cone is obtained by matrix diagonalisation to obtain $\mathbf{E}=$ $\mathbf{R} \boldsymbol{\Lambda} \mathbf{R}^{T}$, where $\mathbf{R}$ is the matrix whose columns are the eigenvectors and $\boldsymbol{\Lambda}=\operatorname{diag}\left[\lambda_{1}, \lambda_{2}, \lambda_{3}\right]$ is the diagonal matrix of corresponding eigenvalues. Applying this rotation to the cone gives the new cone $\lambda_{2} x^{2}+\lambda_{1} y^{2}+\lambda_{3} z^{2}=0$. Recall that a second rotation is then needed to obtain a circle in the image and that this rotation will be about the $x$-axis. Rotation by an angle $\omega$ about the $x$-axis would result in the cone:

$$
\begin{aligned}
\lambda_{2} x^{2}+\left(\lambda_{1} \cos ^{2} \omega+\lambda_{3} \sin ^{2} \omega\right) y^{2}+( & \left.\lambda_{1} \sin ^{2} \omega+\lambda_{3} \cos ^{2} \omega\right) z^{2}+ \\
2\left(\lambda_{1}+\lambda_{3}\right) \sin \omega \cos \omega y z & =0
\end{aligned}
$$

In order to obtain a circle, the $x^{2}$ and $y^{2}$ coefficients must be equated:

$$
\lambda_{2}=\left(\lambda_{1} \cos ^{2} \omega+\lambda_{3}\left(1-\cos ^{2} \omega\right)\right)
$$

Rearranging gives the desired $x$-axis rotation:

$$
\omega= \pm \cos ^{-1} \sqrt{\frac{\lambda_{2}-\lambda_{3}}{\lambda_{1}-\lambda_{3}}}
$$

In general there is a fourfold ambiguity in the value of $\omega$ corresponding to the four quadrants.

In standard clinical practice, the x-ray beam is centred on the symphysis pubis. Since the distance on the x-ray film between the symphysis pubis and the acetabulum is small relative to the film-focus distance, $f$, the beam centre is taken to be at the acetabulum in 
what follows. This simplifying assumption introduces an approximation error. Eccentricity of the rim marker ellipse is then explained in terms of rotation in depth and the eccentricity error's component in the direction of the ellipse's major axis is zero. An expression for the eccentricity error, $\epsilon$, can therefore be computed as the midpoint of the two projected ellipse points that lie on the minor axis (Fig. 17):

$$
\begin{aligned}
\epsilon & =\frac{1}{2}\left(\frac{f r \cos \omega}{d+r \sin \omega}-\frac{f r \cos \omega}{d-r \sin \omega}\right) \\
& =\frac{-f \sin \omega \cos \omega}{\left(\frac{d}{r}\right)^{2}-\sin ^{2} \omega}
\end{aligned}
$$

where $r$ is the radius of the acetabular cup and $d$ is the distance between the acetabular rim centre and the focus. In the experiments reported here, the rotation in depth, $\omega$, was estimated using Equation (19) and assumed to lie in the first quadrant.

\subsection{Empirical Investigation}

A training set of 45 postoperative radiographs containing Zimmer CPT prostheses with $22.225 \mathrm{~mm}$ diameter heads was used. The test set consisted of 50 cases of Zimmer CPT prostheses with $28 \mathrm{~mm}$ head diameter. Each case had year 1 and year 5 radiographs taken consecutively. 175 cases were queried from the patient database. 3 were rejected due to there being no hip prosthesis in one or both the radiographs, 19 were rejected due to the presence of a metal backed acetabular cup, 12 due to eccentricities higher than 0.96 being present in one or both of the radiographs, 6 due to poor exposure in one or both of the radiographs and 1 case due to fatal fracture of the femoral component at year 5 . The test data therefore consisted of $\mathbf{5 0}$ cases chosen uniformly at random from the remaining 134 cases. These were standard clinical radiographs for which the exposure parameters $f$ and $d$ were not recorded. Each radiograph was digitised at 150 dpi. Ellipse models were appropriately scaled to account for the relative size difference of the femoral heads in training and test data.

Given the Monte-Carlo approach to generating five-point subsets for robust parameter estimation, the active ellipses method exhibits variation. The effect on repeatability of varying the number of normals used for active ellipse search was examined. Paired measurements i.e. two measurements were obtained from each radiograph) of the distance between the rim and head ellipse centres were obtained on the 50 test cases. Table 1 summarises the results. In subsequent experiments, 400 normals were used. Other parameter values used in these experiments are detailed in Table 2.

Paired measurements were made of the Euclidean distance between the estimated head and rim centres (without eccentricity correction). There was no failure of fitting. At year 1, the differences between paired measurements of the Euclidean distance between the femoral head and acetabular rim centres were distributed with standard deviation $\sigma=0.09$ and at year 5 with $\sigma=0.07$. In both cases, the mean difference was $0.00 \mathrm{~mm}$. 
Table 1

The effect of varying the number of normals. Means and standard deviations of differences between paired measurements of the inter-centre distance.

\begin{tabular}{c|ccccccc} 
Number of normals & 40 & 80 & 120 & 160 & 200 & 400 & 800 \\
\hline Mean difference (mm) & 0.02 & 0.01 & -0.01 & -0.01 & 0.01 & 0.00 & 0.00 \\
Standard deviation & 0.16 & 0.14 & 0.12 & 0.12 & 0.11 & 0.11 & 0.11
\end{tabular}

Table 2

Parameter values used for active ellipse search.

\begin{tabular}{l|c} 
Parameter & Value \\
\hline Length of model profiles $(k)$ & 31 pixels \\
Length of femoral head search normals $\left(m_{1}\right)$ & 111 pixels \\
Length of rim search normals $\left(m_{2}\right)$ & 61 pixels \\
Convergence criteria for $\Delta a, \Delta b, \Delta x_{c}, \Delta y_{c}$ & 0.1 pixels \\
Convergence criteria for $\Delta \theta$ & $10^{\circ}$ \\
Maximum number of iterations before termination & 30 \\
LSO step size $(\kappa)$ & 1.2 \\
Number of minimal subsamples for LMedS $(h)$ & 300
\end{tabular}

It is worth noting that this demonstrates better repeatability than human annotators tasked with providing point sets for overconstrained ellipse fitting (55). Fig. 18 shows a distribution of wear estimates obtained without eccentricity correction.

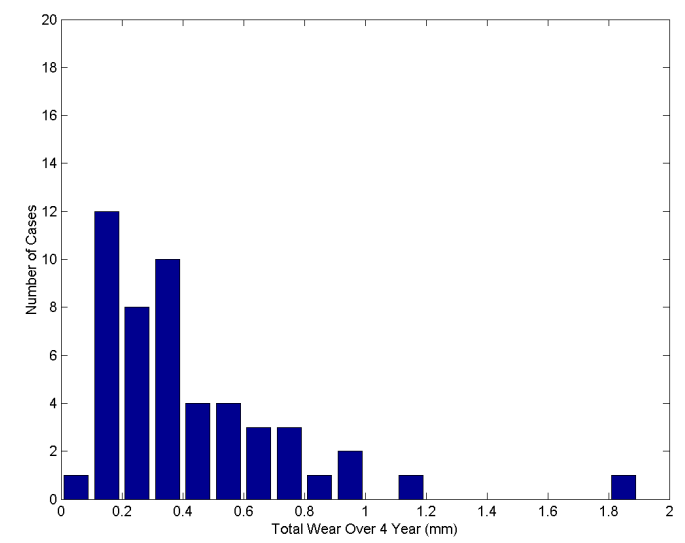

Fig. 18. Distribution of the magnitude of wear vectors over 4 years for the (uncorrected) active ellipses method

Fig. 19 shows the distributions of rotations in depth computed using Equation (19) at year 1 and year 5 represented as histograms with a bin size of $5^{\circ}$. Since values for $f$ and $d$ were unavailable values reported by Krismer et al. (19) were used. Krismer et al. reported minimum, mean and maximum values for $f$ of $900 \mathrm{~mm}, 1000 \mathrm{~mm}$ and $1300 \mathrm{~mm}$ respectively and object-film distances between $180 \mathrm{~mm}$ and $270 \mathrm{~mm}$. A middle value of $225 \mathrm{~mm}$ was used here as an estimate for the expected value. Using the minimum values of $f$ and $d$ would generate the largest error and thus would be a worst case scenario. The maximal values 


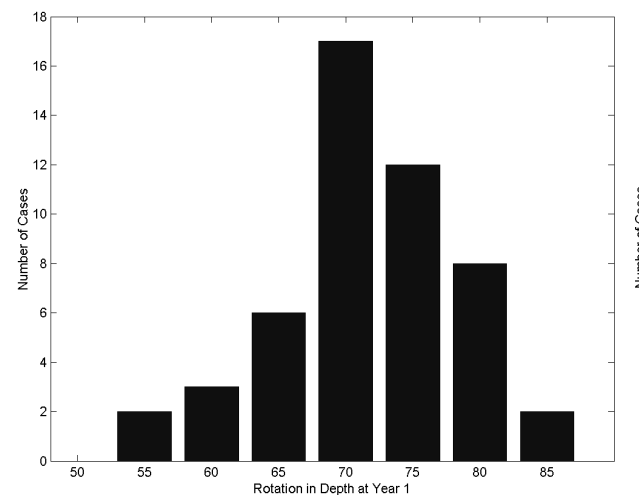

(a)

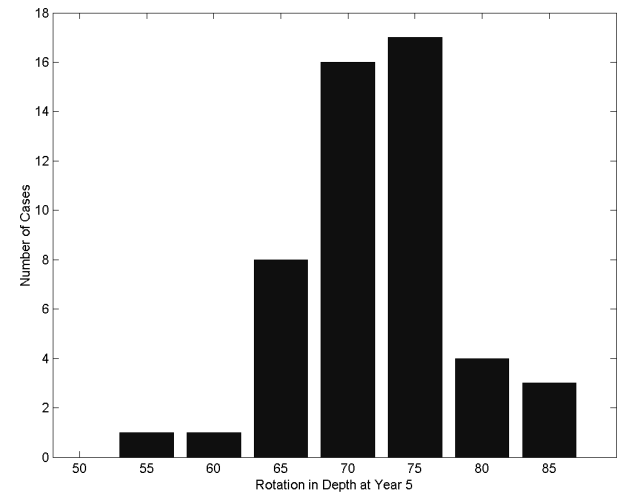

(b)

Fig. 19. Distribution of rotations in depth in degrees obtained for (a) Year 1 and (b) Year 5 radiographs.

would be the best case scenario. The middle values represent the expected case. Fig. 20 illustrates the best, expected and worst case eccentricity errors for one of the radiographs. Displacing the ellipse centre along the minor axis by a distance $\epsilon$ recovers the projection of the rim centre and thus corrects for the eccentricity error.

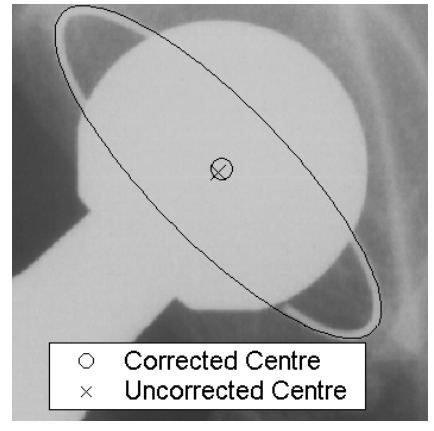

(a)

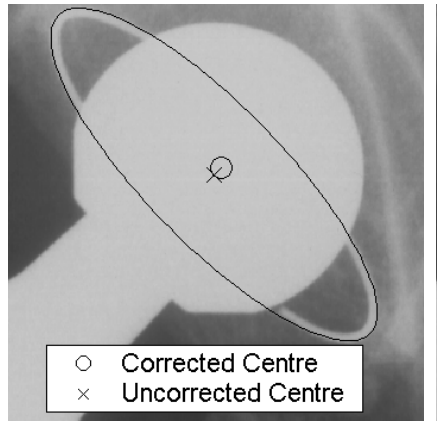

(b)

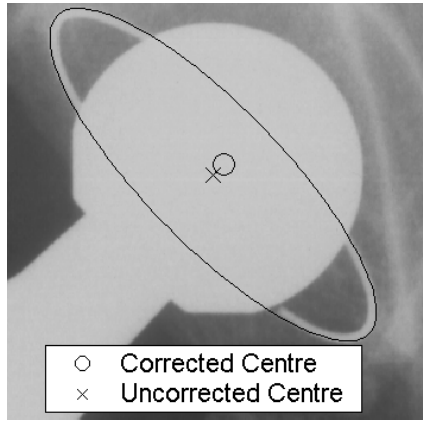

(c)

Fig. 20. Visualisation of correction results where (a) $f=1300 \mathrm{~mm}, d=1120 \mathrm{~mm}$, (b) $f=1000 \mathrm{~mm}, d=775 \mathrm{~mm}$, and (c) $f=900 \mathrm{~mm}, d=630 \mathrm{~mm}$.

If the rim ellipse centre is used in place of the true centre, eccentricity error will affect wear estimates. If the eccentricity error is consistent over time, the wear error could be small. In order to investigate the possibility of such consistency, the absolute difference between rotations in depth at year 1 and year 5 was computed for each case. The resulting distribution is plotted in Fig. 21(a).

The effect of correcting for eccentricity error upon wear estimates was computed using the worst, expected and best case exposure parameters. The distributions of the differences between wear estimates with and without correction are plotted in Fig. 21(b). Standard deviations were found to be $0.016 \mathrm{~mm}, 0.026 \mathrm{~mm}$ and $0.036 \mathrm{~mm}$, with means of $-0.004 \mathrm{~mm}$, $-0.008 \mathrm{~mm}$ and $-0.012 \mathrm{~mm}$ for the the best, expected and worst case exposure parameter values.

Wear estimates were computed as vectors describing the direction of wear for the test set using Equation (13) (without eccentricity correction). There were no cases that had failure of fitting. Fig. 18 shows the distribution of the resulting estimates without eccen- 


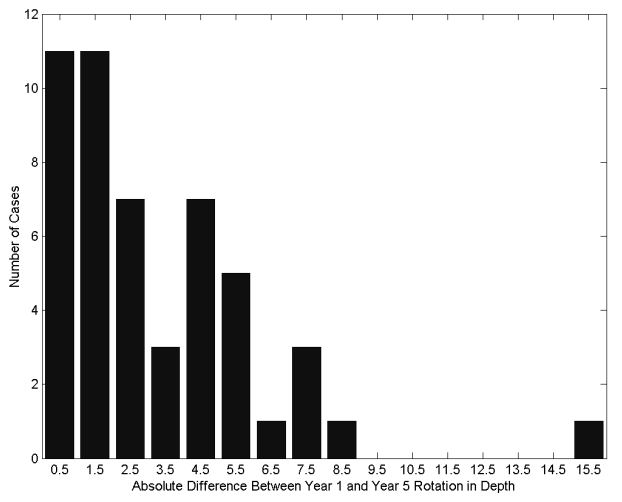

(a)

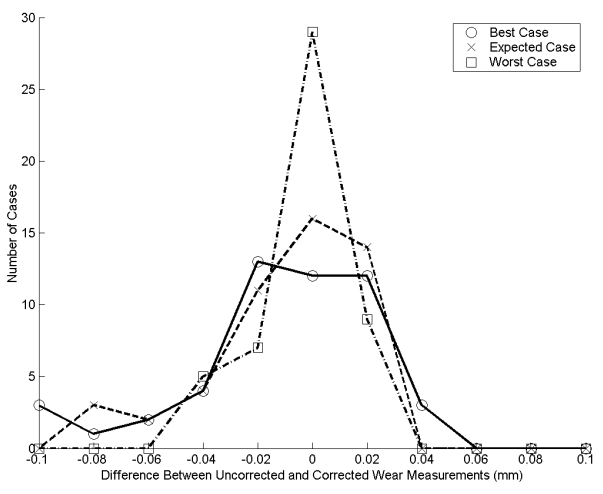

(b)

Fig. 21. (a) Distribution of absolute differences between Year 1 and Year 5 rotations in depth in degrees. (b) Binned distribution of differences between uncorrected and corrected wear values for different exposure parameters. Sample means (standard deviations) of these distributions were $0.00(0.02), 0.00(0.03)$ and $-0.02(0.04)$ for best, expected and worst cases, respectively.

tricity correction and Table 3 gives the means and standard deviations with and without eccentricity corrections. Eccentricity correction has little overall effect on the repeatability of the duo-radiographic method or on the mean of the wear measurements obtained. However the eccentricity error can have a noticeable effect on individual measurements, the maximum errors assuming best, expected and worst case parameters being $0.04 \mathrm{~mm}$, $0.08 \mathrm{~mm}$ and $0.10 \mathrm{~mm}$, respectively.

Table 3

Mean wear estimates (over 4 years) and mean differences between paired wear measurements (standard deviations in parentheses)

\begin{tabular}{c|c|c} 
Method & $\begin{array}{c}\text { Linear wear: } \\
\text { years 1 to 5 (mm) }\end{array}$ & $\begin{array}{c}\text { Mean difference between } \\
\text { paired measurements }(\mathrm{mm})\end{array}$ \\
\hline Uncorrected : ellipse centres & $0.42(0.32)$ & $0.02(0.11)$ \\
Corrected: Best case & $0.43(0.33)$ & $0.01(0.10)$ \\
Corrected: Expected case & $0.43(0.33)$ & $0.02(0.10)$ \\
Corrected: Worst case & $0.44(0.33)$ & $0.02(0.11)$
\end{tabular}

\section{Discussion and Conclusions}

A fully automated method for measuring acetabular wear was described. The only other fully automated method for radiographic analysis of total hip replacements is based on roentgen stereogrammetric analysis (RSA) which has been used for measuring prothesis migration (56). In contrast to RSA, the method presented does not require specialised equipment or invasive apparatus, only a standard PC and x-ray acquisition set-up. This low overhead, coupled with the robustness of fitting and increased repeatability over manual annotation mean that the method holds promise for use in standard clinical settings, although further work will first be needed to estimate the bias of the method. 
The use of robust LMedS fitting with an orthogonal error function was introduced and demonstrated to provide good results when compared to competing methods. In applications where computational speed is important, the foci bisector distance can be recommended as a suitable approximation.

Consistent rotation in depth tends to mitigate the effect on duoradiographic wear measurements of eccentricity error introduced by using the projected acetabular rim centre as a reference point. Nevertheless, the magnitude of this systematic error is sufficient to warrant careful consideration. It can have a significant effect on individual wear measurements producing four year changes as large as $0.04 \mathrm{~mm}, 0.08 \mathrm{~mm}$ and $0.10 \mathrm{~mm}$ assuming best, expected and worst case exposure parameter values, respectively. Linear wear rates have been found experimentally to range between $0.09 \mathrm{~mm} /$ year and $0.25 \mathrm{~mm} /$ year (57) so these errors can become clinically relevant. This serves to highlight the importance of recording $f$ and $d$ at acquisition in order that these sources of error can be controlled. Correcting for this source of error in the proposed method is novel and should also be applied to other methods that use the centre of the rim. Examples of these methods include EBRA (19) and Eggli et al. (37) when using the centre of the acetabular rim as a reference point. The effect of eccentricity error on uniradiographic methods will be more pronounced.

Eccentricity error due to translation of the acetabular cup away from the beam centre was not considered in this study. Such translation introduces further error including, in general, a component in the direction of the ellipse's major axis. In standard clinical radiographs the beam centre often lies on the symphysis pubis which can be as much as 900 pixels away from the acetabular rim centre on a 150 dpi radiograph. If the location of the beam centre, or that of the symphysis pubis as an approximation to the beam centre, were known, the full eccentricity error could be estimated. Ahn et al. (58) provide a detailed treatment of the relevant geometry (using a camera model).

\section{Acknowledgments}

S. Kerrigan was funded by Zimmer Ltd. The authors are grateful to Elizabeth Berry (University of Leeds) for helpful comments on aspects of this work, to Lynda Cochrane for her help with the statistics and to Tony Mochan and Jeremy Martindale for their help in assembling the dataset.

\section{References}

[1] M. Huo, S. Cook, Speciality update: What's new in hip arthroplasty, Journal of Bone and Joint Surgery 83-A (2001) 1598-1610.

[2] M. Olmstead, Canine cemented total hip replacements: state of the art, Journal of Small Animal Practice 36 (1995) 395-399.

[3] A. Furnes, S. Lie, L. Havelin, L. Engesaeter, S. Vollset, The economic impact of failures in total hip replacement, Acta Orthopaedics Scandinavia 67 (1985) 115-121. 
[4] A. Murray, D.W. Carr, C. Bulstrode, Which primary total hip replacement?, Journal of Bone and Joint Surgery 77-B (1995) 520-527.

[5] W. Harris, Hybrid total hip replacement: rationale and intermediate clinical results, Clinical Orthopaedics 333 (1996) 155-164.

[6] B. Wroblewski, 15-21 year results of the charnley low-friction arthroplasty, Clinical Orthopaedics and Related Research 211 (1986) 30-35.

[7] A. Blom, A. Taylor, G. Pattison, S. Whitehouse, G. Bannister, Infection after total hip arthroplasty, Journal of Bone and Joint Surgery 85-A (2003) 956-959.

[8] H. Malchau, P. Herberts, T. Eisler, G. Garellick, P. Soderman, The swedish total hip replacement register, Journal of Bone and Joint Surgery 84-A Suppl 2 (2002) 2-20.

[9] H. McKellop, F. Shen, B. Lu, P. Campbell, R. Salovey, Development of an extremely wear-resistant ultra high molecular weight polyethylene for total hip replacements, Journal of Orthopaedic Research 17 (1999) 157-167.

[10] D. Howie, B. Vernon-Roberts, R. Oakeshott, B. Manthey, A rat model of resorption of bone at the cement-bone interface in the presence of polyethylene wear particles, Journal of Bone and Joint Surgery 70-A (1988) 254-258.

[11] Y. Zhu, K. Chiu, W. Tang, Review article: Polyethylene wear and osteolysis in total hip arthroplasty, Journal of Orthopaedic Surgery 9(1) (2001) 91-99.

[12] H. Cates, P. Faris, E. Keating, M. Ritter, Polyethylene wear in cemented metalbacked acetabular cups, Journal of Bone and Joint Surgery 75-B (1993) 249-257.

[13] A. Greenwald, J. Garino, Alternative bearing surfaces: The good, the bad and the ugly, Journal of Bone and Joint Surgery 83-A (2001) 68-72.

[14] J. Livermore, D. Ilstrup, B. Morrey, Effect of femoral size on wear of the polyethylene acetabular components, Journal of Bone and Joint Surgery 72-A (1990) 518-528.

[15] J. Kabo, J. Gebhard, G. Loren, H. Amstutz, In vivo wear of polyethylene acetabular components, Journal of Bone and Joint Surgery 75-B (1993) 254-258.

[16] J. Collier, M. Mayor, V. Surprenant, L. Dauphinais, R. Jensen, The biomechanical problems of polyethylene as a bearing surface, Clinical Orthopaedics and Related Research 261 (1990) 107-113.

[17] R. Looney, B. Boyd, S. Totterman, G. Seo, J. Tamez-Pana, D. Campbell, L. Novotny, C. Olcott, J. Martell, F. Hayes, R. O'Keefe, E. Schwarz, Volumetric computerized tomogrophy as a measurement of prosthetic acetabular osteolysis and its correlation with wear, Arthritis Research 4 (2002) 59-63.

[18] K. Burckhardt, C. Gerber, J. Hodler, N. H., G. Szekely, Precision of distance determination using $3 \mathrm{~d}$ to $2 \mathrm{~d}$ projections: The error of migration measurement using x-ray images, Medical Image Analysis 4 (2000) 375-388.

[19] M. Krismer, R. Bauer, J. Tschupik, P. Mayrhofer, EBRA: A method to measure migration of acetabular components, Journal of Biomechanics 28 (1995) 1225-1236.

[20] D. Nunn, M. Freeman, P. Hill, S. Evans, The measurement of migration of the actebular component of hip prostheses, Journal of Bone and Joint Surgery 71-B (1989) 629-631.

[21] A. J. Hui, R. W. McCalden, J. M. Martell, S. J. MacDonald, R. B. Bourne, C. H. Rorabeck, Validation of two and three-dimensional radiograph techniques for measuring polyethelyne wear after total hip replacement, Journal of Bone and Joint Surgery 85-A (2003) 505-511.

[22] J. Charnley, D. Halley, Rate of wear in total hip replacement, Clinical Orthopaedics and Related Research 112 (1975) 170-179. 
[23] J. Charnley, Z. Cubic, Nine and ten year results of low-friction arthroplasty of the hip, Clinical Orthopaedics and Related Research 95 (1973) 9-25.

[24] J. Clarke, K. Black, C. Rennie, H. Amsutz, Can wear in total hip replacements be assessed from radiographs, Clinical Orthopaedics and Related Research (1976) 126142.

[25] M. Griffith, M. Seidenstein, D. Williams, J. Charnley, Socket wear in charnley low friction arthroplasty of the hip, Clinical Orthopaedics and Related Research 137 (1978) 37-47.

[26] B. Wroblewski, Direction and rate of socket wear in charnley low-friction arthroplasty, Journal of Bone and Joint Surgery 67-B (1985) 757-761.

[27] J. Martell, S. Berdia, Determination of polyethylene wear in total hip replacements with use of digital radiographs, Journal of Bone and Joint Surgery 79-A (1997) 16351641.

[28] E. Otterberg, D. Hauser, M. Siddiqui, C. Bragdon, W. Harris, Radiographic techniques to assess in vivo polyethylene wear following total hip arthroplasty, Harvard Orthopaedic Journal 2.

[29] H. Cowell, Editorial: Radiographic measurements and clinical decisions, Journal of Bone and Joint Surgery 72-A (1990) 319.

[30] P. Jones, C. Taylor, D. Hukins, K. Hardinge, M. Porter, Prosthetic hip failure: Preliminary findings of retrospective radiograph image analysis, Enginnering in Medicine 17 (1988) 119-125.

[31] K. Hardinge, M. Porter, P. Jones, D. Hukins, C. Taylor, Measurement of hip prostheses using image analysis: The maxima technique, Journal of Bone and Joint Surgery 73-B (1991) 724-728.

[32] A. Redhead, A. Kotcheff, C. Taylor, M. Porter, D. Hukins, An automated method for assessing routine radiographs of patients with total hip replacements, Proceedings of the Institution of Mechanical Engineers 211 (1997) 145-154.

[33] S. Shaver, T. Brown, S. Hillis, J. Callaghan, Digital edge-detection measurement of polyethylene wear after total hip arthroplasty, Journal of Bone and Joint Surgery 79-A (1997) 690-700.

[34] A. Fitzgibbon, M. Pilu, R. Fisher, Direct least square fitting of ellipses, IEEE Transactions on Pattern Analysis and Machine Intelligence 21 (5) (1999) 476-480.

[35] K. Chen, J. Wu, Measurement of polyethylene wear - a new three-dimensional methodology, Computer Methods and Programs in Biomedecine 68 (2002) 117-127.

[36] F. Hatfield, R. Hall, R. King, E. Berry, P. Siney, B. Wroblewski, Image analysis of wear in total hip replacements, Proceedings of Medical Image Understanding and Analysis.

[37] S. Eggli, S. Z'Brun, C. Gerber, R. Ganz, Comparison of polyethylene wear with femoral heads of $22 \mathrm{~mm}$ and $32 \mathrm{~mm}$. a prospective randomised study, Journal of Bone and Joint Surgery 137 (1978) 447-447.

[38] Z. Knudsen, R. Hall, E. Berry, P. Siney, B. Wroblewski, Assessment of digital image processing in the measurement of wear in cemented thrs, Journal of Bone and Joint Surgery Supp II-B (2001) 135.

[39] P. Devane, R. Bourne, C. Rorabeck, R. Hardie, J. Horne, Measurement of polyethylene wear in metal-backed acetabular cups, Clinical Orthopaedics and Related Research 319 (1995) 303-316.

[40] T. Cootes, C. Taylor, Statistical models of appearance for medical image analysis and 
computer vision, Proceedings of the SPIE Medical Imaging 4322 (2001) 236-248.

[41] R. Halir, J. Flusser, Numerically stable direct least squares fitting of ellipses. URL citeseer.nj.nec.com/350661.html

[42] P. Rosin, Assessing error of fit functions for ellipses, Graphical models and image processing: GMIP 58 (5) (1996) 494-502.

URL citeseer.nj.nec.com/rosin96assessing.html

[43] P. Rosin, Analyzing error of fit functions for ellipses, Pattern Recognition Letters 17 (1996) 1461-1470.

[44] S. J. Ahn, W. Rauh, H. J. Warnecke, Least squares orthogonal distances fitting of circle, sphere, ellipse, hyperbola and parabola, Pattern Recognition 34 (2001) 22832303.

[45] S. J. Ahn, W. Rauh, H. S. Cho, H. Warnecke, Orthogonal distance fitting of implicit curves and surfaces, IEEE Transactions on Pattern Analysis and Machine Intelligence 24 (5) (2002) 620-638.

[46] J. Huber, Robust Statistics, Wiley, 1981.

[47] G. Roth, M. Levine, Extracting geometric primitives, CVGIP: Image Understanding 58 (1991) 1-22.

[48] Z. Zhang, Parameter estimation techniques: A tutorial with respect to conic fitting, Image and Vision Computing 15 (1997) 59-76.

[49] M. Rogers, J. Graham, Robust active shape model search, 7th European Conference on Computer Vision 4 (2002) 517-530.

[50] M. Rogers, J. Graham, Robust active shape model search for medical image analysis, Proceedings of the MIUA (2002) 81-84.

[51] P. J. Rousseeuw, A. M. Leroy, Robust Regression and Outlier Detection, Wiley, 2003.

[52] P. Rosin, Further five-point fit ellipse fitting, Graphical Models and Image Processing 61 (5) (1999) 245-259.

[53] R. Safaee-Rad, I. Tchoukanov, B. Benhabib, K. Smith, Accurate parameter estimation of quadratic curves from grey-level images, CVGIP: Image Understanding 54 (1991) 259-274.

[54] D. Forsyth, J. Mundy, A. Zisserman, C. Coelho, A. Heller, C. Rothwell, Invariant descriptors for 3-d object recognition and pose, IEEE Transactions on Pattern Analysis and Machine Intelligence 13 (10) (1991) 971-991.

[55] S. Kerrigan, S. J. McKenna, I. W. Ricketts, C. A. Wigderowitz, Analysis of total hip replacements using active ellipses, in: Medical Image Understanding and Analysis, Sheffield, 2003, pp. 177-180.

[56] J. Karrholm, Roentgen stereophotogrammetry: An overview of orthopedic applications, Acta Orthop Scand 60 (1989) 491-503.

[57] R. Kosak, V. Antolic, V. Pavlovcic, V. Karlj-Iglic, I. Milosev, G. Vidmar, A. Iglic, Polyethylene wear in total hip prostheses: The influence of direction of linear wear on volumetric wear determined from radiographic data, Journal of Skeletal Radiology 32 (2003) 679-686.

[58] S. Ahn, H.-J. Warnecke, R. Kotowski, Systematic geometric image measurement errors of circular object targets: Mathematical formulation and correction, Photogrammetric Record 16 (93) (1999) 485-502. 